\title{
Resonant scattering on impurities in the Quantum Hall Effect
}

\author{
S. A. Gurvitz \\ Department of Nuclear Physics, Weizmann Institute of Science, Rehovot 76100, Israel \\ and TRIUMF, Vancouver, B.C., Canada V6T $2 A 3$
}

\begin{abstract}
We develop a new approach to carrier transport between the edge states via resonant scattering on impurities, which is applicable both for short and long range impurities. A detailed analysis of resonant scattering on a single impurity is performed. The results are used for study of the inter-edge transport by multiple resonant hopping via different impurities' sites. It is shown that the total conductance can be found from an effective Schroedinger equation with constant diagonal matrix elements in the Hamiltonian, where the complex non-diagonal matrix elements are the amplitudes of a carrier hopping between different impurities. It is explicitly demonstrated how the complex phase leads to Aharonov-Bohm oscillations in the total conductance. Neglecting the contribution of self-crossing resonant-percolation trajectories, one finds that the inter-edge carrier transport is similar to propagation in onedimensional system with off-diagonal disorder. We demonstrated that each Landau band has an extended state $\bar{E}_{N}$, while all other states are localized. The localization length behaves as $L_{N}^{-1}(E) \sim\left(E-\bar{E}_{N}\right)^{2}$.
\end{abstract}




\section{INTRODUCTION}

The quantum Hall effect (QHE) is characterized by plateaus of zero longitudinal resistance and quantized Hall resistance as a function of the magnetic field. When the Fermi level passes through the plateaus, the QHE breaks down, and the longitudinal resistance appears. The explanation of the integer QHE in a the framework of the Landauer approach [1] is based on the suppression of backscattering in a strong magnetic field [2]: a carrier moving along the boundary of a sample cannot reverse direction of its motion if scattered by an impurity, unless it jumps to another boundary. If a sample is much wider then the magnetic length, the inter-edge scattering is likely to be negligible and the conductance is expected to be quantized. However, when the Fermi energy is near Landau level centers, the electron states extend across the sample. As a result, the inter-edge current appears and so does the longitudinal resistance.

The localization and scaling properties of a disordered 2D electron gas near the Landau level in the presence of a strong external magnetic field has been a subject of many numerical [3] and analytical studies [4]. Although various computer simulations strongly support the concept of the power-law divergence of the localization length near the Landau level center [3], the question as to the nature of the delocalization is not yet resolved.

Actually, the strong-field Landau localization is related to the $2 \mathrm{D}$ scattering problem on randomly distributed impurities. The problem can be essentially simplified if the electronimpurity scattering goes through quasi-stationary states near impurity sites. In fact, this always happens when the electron energy passes the Landau levels, since the impurities generate quasi-stationary states near the Landau levels thresholds. In this case one can retain only the resonance part in the electron-impurity scattering amplitude, instead of solving the $2 \mathrm{D}$ scattering problem in its full complexity.

We concentrate in this paper on the resonant scattering on impurities in QHE in general, and in connection with the localization properties of electronic states near Landau levels. For treatment of this problem we propose a new approach, which is based on couple- 
channel multiple-scattering theory and allows an analysis of QHE both in narrow and in wide samples. This approach reduces $2 \mathrm{D}$ scattering problem to an effectively $1 \mathrm{D}$ problem, and therefore makes it possible to investigate the localization and scaling properties of a distorted 2D system analytically.

We start with a detailed investigation of the inter-edge transitions via the resonance scattering on a single impurity. Although this process has been discussed in the literature [5 8], the analysis was always concentrated on a specific type of the impurity potentials. Here we present an unified approach and derive simple analytical expressions for the resonance energy and the partial widths. However, the probability of such direct enter-edge transitions drops down very rapidly with a sample width. Hence, the resonant tunneling on a single impurity can produce an appreciable effect only in narrow samples. One can expect that in a the case of wide samples the inter-edge resonant transport goes via by multiple resonant transitions (hopping) of a carrier via different impurities. In fact, this process had not attracted an appropriate attention, except for a similar problem of tunneling transparency of disordered systems (with no magnetic field), which has been studied by Lifshitz and Kirpichenkov [9]. In the second part of the paper we concentrate on $2 \mathrm{D}$ electron gas transport via subsequent resonant scattering on different impurities in the presence of a strong magnetic field. The electron-electron interaction is not considered here.

The paper is organized as follows: General description of the couple-channel approach is given in Section 2. In Section 3 we present a detailed analysis of resonant scattering on a single impurity. The multiple resonance scattering on two impurities is discussed in Section 4. We singled out this particular case in order to exemplify how our approach is extended to any number of impurities participating in the resonant transport. We also obtain there an analytical expression for the complex (off-diagonal) amplitude describing the resonant hopping between different impurity sites and for the diagonal energy shift. The resonant transport via $n$ impurities is discussed in Section 5. Section 6 is the summary. In the last Section we compare our results for localization of the 2D disordered electron gas in a strong magnetic field with those obtained by different methods. 


\section{FORMULATION OF THE PROBLEM}

Consider two-dimensional noninteracting electrons gas in the presence of a perpendicular magnetic field $\boldsymbol{B}$. The electrons are confined along the $y$-direction and free to move along the $x$-direction. Consider also impurities inside the system. The Schroedinger equation describing the electron motion in the $(x, y)$-plane for the Landau gauge, $\boldsymbol{A}=(-B y, 0,0)$, is

$$
\left[\frac{1}{2 m}\left(-i \hbar \frac{d}{d x}+\frac{e}{c} A_{x}\right)^{2}-\frac{\hbar^{2}}{2 m} \frac{d^{2}}{d y^{2}}+W(y)+V(x, y)\right] \Psi(x, y)=E \Psi(x, y)
$$

where $m$ is the electron effective mass. The potential $W(y)$ provides confinement of the

electron motion along the $y$-direction. For the definiteness we take it in a form of confining walls, $W(y)=0$ for $|y| \leq a$ and $W(y)=+\infty$ for $|y|>a$. The impurities inside the channel are described by the potential $V(x, y) \equiv \sum_{j} V_{j}\left(x-x_{j}, y-y_{j}\right)$, where $\left(x_{j}, y_{j}\right)$ are the coordinates of the impurity centers.

In the absence of impurities $(V=0)$ the solution of Eq. (2.1) can be written as

$$
\phi_{n k}(x, y)=e^{i k x} \Phi_{n k}(y)
$$

where $\Phi_{n k}$ satisfies the reduced equation

$$
\left[-\frac{\hbar^{2}}{2 m} \frac{d^{2}}{d y^{2}}+\frac{m \omega_{c}^{2}\left(y-\ell^{2} k\right)^{2}}{2}+W(y)\right] \Phi_{n k}(y)=E_{n k} \Phi_{n k}(y)
$$

with $\omega_{c}=e B / m c$ is the cyclotron frequency, $\ell=\sqrt{\hbar c / e B}$ is the magnetic length and

$$
E_{n k}=E_{n}+\mathcal{K}_{n}(k)
$$

The energy spectrum $E_{n k}$, illustrated schematically in Fig. 1, depends on the continuum wave vector $k$ and the Landau mode index $n$. If there are no confining walls, $W(y)=0$, one finds that $E_{n}=(n+1 / 2) \hbar \omega_{c}, \mathcal{K}_{n}(k)=0$, and $\Phi_{n k}(y)$ are the harmonic oscillator wave functions

$$
\Phi_{n k}(y) \equiv \Phi_{n}\left(y-\ell^{2} k\right)=\frac{1}{\pi^{1 / 4}\left(2^{n} \ell n !\right)^{1 / 2}} H_{n}\left(\frac{y-\ell^{2} k}{\ell}\right) \exp \left(-\frac{\left(y-\ell^{2} k\right)^{2}}{2 \ell^{2}}\right)
$$


In the presence of confining walls the Landau-mode wave functions, $\Phi_{n k}(y)$, are given in terms of parabolic cylinder functions. For $\bar{a} \equiv a / \ell \gg n$ and $k \ell \ll \bar{a}$, one can use the asymptotic expansion of parabolic cylinder functions (Appendix A) thus obtaining $E_{n}=$ $\left(n+1 / 2+\Delta_{n}\right) \hbar \omega_{c}$, and

$$
\begin{aligned}
\mathcal{K}_{n}(k) & =\frac{2^{n}}{\sqrt{\pi} n !}\left[(\bar{a}+k \ell)^{2 n+1} e^{-(\bar{a}+k \ell)^{2}}+(\bar{a}-k \ell)^{2 n+1} e^{-(\bar{a}-k \ell)^{2}}-\Delta_{n}\right] \hbar \omega_{c} \\
& \cong \frac{2^{n+2}}{\sqrt{\pi} n !} \bar{a}^{2 n+3} e^{-\bar{a}^{2}}(k \ell)^{2} \hbar \omega_{c}, \quad \text { for } k \ell \ll 1
\end{aligned}
$$

where $\Delta_{n}=2 \bar{a}^{2 n+1} \exp \left(-\bar{a}^{2}\right)$ is the Landau level shift due to the confining walls (this tiny shift will be neglected in the following). One also obtains that the wave functions $\Phi_{n k}$ are well approximated in this region by the harmonic oscillator wave functions: $\Phi_{n k}(y)=\Phi_{n}\left(y-\ell^{2} k\right)$.

It follows from Eqs. (2.5), (2.6) that the carrier velocity $v_{n}=\hbar^{-1} d E_{n}(k) / d k \approx 0$ except for $k \sim \pm a / \ell^{2}$ and $y \sim \pm a$. Thus only the edge states provide the carriers flow in a strong magnetic field. Classically these states correspond to electron orbits hopping along the boundaries of a sample, Fig. 2. Since the velocity $v_{n}$ is positive at the upper edge and negative at the lower edge, the carriers can reverse the direction of motion only by jumping to the opposite edge [2]. This process can be generated by multiple scattering on impurities, Fig. 2, and will be treated as follows.

Consider the general solution of Eq. (2.1), which can be written as

$$
\Psi(x, y)=\phi_{n k}(x, y)+\int G\left(E ; x, y ; x^{\prime}, y^{\prime}\right) V\left(x^{\prime}, y^{\prime}\right) \Psi\left(x^{\prime}, y^{\prime}\right) d x^{\prime} d y^{\prime}
$$

where the Green's function $G$ is

$$
G\left(E ; x, y ; x^{\prime}, y^{\prime}\right)=\sum_{n^{\prime}} \int \frac{e^{i k^{\prime}\left(x-x^{\prime}\right)} \Phi_{n^{\prime}, k^{\prime}}(y) \Phi_{n^{\prime}, k^{\prime}}^{*}\left(y^{\prime}\right)}{E-\left(n^{\prime}+\frac{1}{2}\right) \hbar \omega_{c}-\mathcal{K}_{n^{\prime}}\left(k^{\prime}\right)} d k^{\prime}
$$

The wave function $\phi_{n k}(x, y)$ describes the motion of carriers inside the Landau mode $n$ and incident from the left $(x \rightarrow-\infty)$. It follows from Eqs. (2.7), (2.8) that the full solution, $\Psi(x, y) \equiv \Psi_{n, k_{n}}(x, y)$ at $x \rightarrow-\infty$ can be written as

$$
\Psi_{n, k_{n}}(x, y)=\sum_{n^{\prime}}\left[\delta_{n n^{\prime}} e^{i k_{n} x} \Phi_{n, k_{n}}(y)+\left(v_{n} / v_{n^{\prime}}\right)^{1 / 2} \mathrm{r}_{n n^{\prime}} e^{-i k_{n^{\prime}} x} \Phi_{n^{\prime},-k_{n^{\prime}}}(y)\right]
$$


and the same solution at $x \rightarrow+\infty$ is

$$
\Psi_{n, k_{n}}(x, y)=\sum_{n^{\prime}}\left(v_{n} / v_{n^{\prime}}\right)^{1 / 2} \mathrm{t}_{n n^{\prime}} e^{i k_{n^{\prime}} x} \Phi_{n^{\prime}, k_{n^{\prime}}}(y)
$$

where $k_{n}$ correspond to poles in the denominator of Eq. (2.8):

$$
\mathcal{K}_{n}\left(k_{n}\right)=E-\left(n+\frac{1}{2}\right) \hbar \omega_{c}
$$

Here $\mathrm{r}_{n n^{\prime}}$ and $\mathrm{t}_{n n^{\prime}}$ are the carrier reflection and transmission probability amplitudes for the transition from the mode $n$ into the mode $n^{\prime}$. The corresponding reflection and transmission matrices are $R_{n n^{\prime}}=\left|\mathrm{r}_{n n^{\prime}}\right|^{2}$ and $T_{n n^{\prime}}=\left|\mathrm{t}_{n n^{\prime}}\right|^{2}$. These quantities determine the longitudinal and the Hall resistance. In the following we consider only the total resistance $R=\mathcal{G}^{-1}$, where $\mathcal{G}$ is the two-terminal conductance given by the Landauer formula (in units of $e^{2} / h$ ) 10

$$
\mathcal{G}=\sum_{n, n^{\prime}} T_{n n^{\prime}}
$$

One finds from Eq. (2.8) that for a given energy $\left(E=E_{F}\right)$ there exist two kinds of modes: conducting modes, $n+1 / 2<E / \hbar \omega_{c}$, whose wave numbers are real, and evanescent modes, $n+1 / 2>E / \hbar \omega_{c}$, whose wave numbers are imaginary. The evanescent modes correspond to virtual states and are not propagating. Hence, $n, n^{\prime}<\left(E / \hbar \omega_{c}\right)-1 / 2$ in Eq. (2.12). In a case of weak impurity potential $V$ the coupling between different propagating modes is small, so that the non-diagonal terms $\left(n=n^{\prime}\right)$ in the Landauer formula can be neglected. Also the coupling between propagating and evanescent modes is small. As a result $\mathcal{G}=N$ (the total number of propagating modes participating in the transport), except for the Landau levels regions, $E \sim \hbar \omega_{c}(n+1 / 2)$, where the influence of impurities is resonantly enhanced.

In the following we are going to analyze the resonant scattering near in vicinity of Landau levels using technique of the multichannel scattering theory. This approach has been applied for scattering on impurities in narrow channels with no magnetic field [1] . In the case of QHE the multichannel treatment is more complicated, since the Landau-mode wave functions $\Phi_{n k}(y)$ depend on the wave vector $k$. However, the problem can be technically sim- 
plified if we turn to the mixed, momentum-coordinate representation of the wave function, namely $\Psi(x, y) \rightarrow \tilde{\Psi}(p, y)$, where

$$
\tilde{\Psi}(p, y)=\int \Psi(x, y) e^{-i p x} d x
$$

In this representation the Schroedinger equation (2.1) becomes

$$
\left[E-\frac{1}{2 m}\left(\hbar p-\frac{e}{c} B y\right)^{2}+\frac{\hbar^{2}}{2 m} \frac{d^{2}}{d y^{2}}-W(y)\right] \tilde{\Psi}(p, y)=\int \tilde{V}\left(p-p^{\prime}, y\right) \tilde{\Psi}\left(p^{\prime}, y\right) \frac{d p^{\prime}}{2 \pi}
$$

with

$$
\tilde{V}\left(p-p^{\prime}, y\right)=\int V(x, y) e^{-i\left(p-p^{\prime}\right) x} d x
$$

and $\phi_{n k}(x, y) \rightarrow \tilde{\phi}_{n k}(p, y)$ in Eq. (2.2), which now reads

$$
\tilde{\phi}_{n k}(p, y)=(2 \pi) \delta\left(p-k_{n}\right) \tilde{\Phi}_{n}(p, y)
$$

where the wave vector $k_{n}$ is given by Eq. (2.11).

In order to find the reflection and transmission amplitudes it is useful to expand the full wave function $\tilde{\Psi}(p, y)$, Eq. (2.14), in terms of the Landau mode wave functions $\tilde{\Phi}_{n}$

$$
\tilde{\Psi}(p, y)=\sum_{n} \psi_{n}(p) \tilde{\Phi}_{n}(p, y)
$$

Substituting Eq. (2.17) into Eq. (2.14), multiplying it by $\Phi_{n^{\prime}}^{*}(p, y)$ and integrating over $y$ by use of $\left\langle\tilde{\Phi}_{n p} \mid \tilde{\Phi}_{n^{\prime} p}\right\rangle=\delta_{n n^{\prime}}$, we obtain the system of coupled equations for the wave functions, $\psi_{n}$

$$
\mathcal{K}_{n}(p) \psi_{n}(p)+\sum_{n^{\prime}} \int V_{n n^{\prime}}\left(p, p^{\prime}\right) \psi_{n^{\prime}}\left(p^{\prime}\right) \frac{d p^{\prime}}{2 \pi}=\left(E-E_{n}\right) \psi_{n}(p)
$$

where $E_{n}=(n+1 / 2) \hbar \omega_{c}$, and

$$
V_{n n^{\prime}}\left(p, p^{\prime}\right)=\int \tilde{\Phi}_{n^{\prime}}^{*}\left(p^{\prime}, y\right) \tilde{V}\left(p-p^{\prime}, y\right) \tilde{\Phi}_{n}(p, y) d y=\sum_{j} \bar{V}_{n n^{\prime}}^{j}\left(p, p^{\prime}\right) e^{-i\left(p-p^{\prime}\right) x_{j}}
$$

is the impurity potential projected into the Landau-mode wave functions space. Here

$$
\bar{V}_{n n^{\prime}}^{j}\left(p, p^{\prime}\right)=\int \tilde{\Phi}_{n^{\prime}}^{*}\left(p^{\prime}, y\right) V_{j}\left(x, y-y_{j}\right) \tilde{\Phi}_{n}(p, y) e^{-i\left(p-p^{\prime}\right) x} d x d y
$$


Notice that $V_{n n^{\prime}}\left(p, p^{\prime}\right)$ is a non-local potential, since it depends on the momenta $p$ and $p^{\prime}$ taken separately, although the "original" impurity potential, $V(x, y)$ (or $\tilde{V}\left(p-p^{\prime}, y\right)$, Eq. (2.15) is a local one.

Consider for the definiteness the electron energy $E$ within the range of $E_{0} \leq E \leq E_{1}$. Then this case only the $n=0$ Landau-mode is the propagating mode, whereas all the other modes are evanescent. In general, one can neglect the affect of evanescent modes on carriers propagation in a strong magnetic field. However, when the electron energy $E$ approaches the nearest evanescent Landau mode $(n=1)$, its influence can be important. We thus keep two terms, $n=0,1$ in Eq. (2.17), so that the coupled equations for the first two modes are

$$
\begin{aligned}
& \mathcal{K}_{0}(p) \psi_{0}(p)+\int V_{00}\left(p, p^{\prime}\right) \psi_{0}\left(p^{\prime}\right) \frac{d p^{\prime}}{2 \pi}+\int V_{01}\left(p, p^{\prime}\right) \psi_{1}\left(p^{\prime}\right) \frac{d p^{\prime}}{2 \pi}=\left(E-E_{0}\right) \psi_{0}(p) \\
& \mathcal{K}_{1}(p) \psi_{1}(p)+\int V_{11}\left(p, p^{\prime}\right) \psi_{1}\left(p^{\prime}\right) \frac{d p^{\prime}}{2 \pi}+\int V_{10}\left(p, p^{\prime}\right) \psi_{0}\left(p^{\prime}\right) \frac{d p^{\prime}}{2 \pi}=\left(E-E_{1}\right) \psi_{1}(p)
\end{aligned}
$$

It is useful to introduce the channel-mode Green's functions

$$
\begin{aligned}
& G_{0}(E)=\left(E-E_{0}-\mathcal{K}_{0}-V_{00}\right)^{-1}, \\
& G_{1}(E)=\left(E-E_{1}-\mathcal{K}_{1}-V_{11}\right)^{-1},
\end{aligned}
$$

which describe one-dimensional motion inside the Landau modes $n=0$ and $n=1$ respectively. Then one gets from Eqs. (2.21b)

$$
\psi_{1}=G_{1} V_{10} \psi_{0}
$$

Substituting Eq. (2.23) into Eq. (2.21a) one obtains the following equation for $\psi_{0}$

$$
\left(E-E_{0}-\mathcal{K}_{0}-V_{00}\right) \psi_{0}=V_{01} G_{1}(E) V_{10} \psi_{0}
$$

Eq. (2.24) is the one-dimensional equation describing a carrier propagating inside the mode $n=0$. If $V=0$ (no impurities), then $\psi_{0}(p) \rightarrow 2 \pi \delta\left(p \pm k_{0}\right)$ which corresponds to free electron motion near the upper or the lower edge with the velocity $v_{0}^{ \pm}=\hbar^{-1}\left[d \mathcal{K}_{0}(p) / d p\right]_{p= \pm k_{0}}$ respectively. The impurity potential $V$ generates carriers scattering. The appropriate penetration amplitude, $\mathrm{t}_{00}$, is obtained from $\psi_{0}(p)$ taken in the asymptotic regions, Eq. (2.10), 
which corresponds to $p \rightarrow \pm k_{0}$ in the $p$-representation. Respectively, the total conductance $\mathcal{G}=\left|t_{00}\right|^{2}$, Eq. (2.12).

Using the above approach we are going to investigate resonant transitions between the edges, generated by an interaction between carriers and impurities. We start with a detailed analysis of the resonant scattering on a single impurity in the threshold regions, $E \sim E_{n}$. For the definiteness we consider the electron energy within the range of $E_{0} \leq E \leq E_{1}$, where only the Landau mode $n=0$ is the propagating one, though the same treatment is valid in a general case.

\section{RESONANT SCATTERING ON A SINGLE IMPURITY}

\section{A. Attractive impurity}

Consider Eq. (2.24) near the $n=1$ Landau mode threshold, $E \lesssim E_{1}$. Let us assume that the operator $\mathcal{K}_{1}+V_{11}$ in the Green's function $G_{1}(E)$, Eq. (2.22b), has at least one eigenstate in the discrete spectrum, i.e.

$$
\mathcal{K}_{1}(p) \tilde{\varphi}_{1}(p)+\int V_{11}\left(p, p^{\prime}\right) \tilde{\varphi}_{1}\left(p^{\prime}\right) \frac{d p^{\prime}}{2 \pi}=\epsilon_{1} \tilde{\varphi}_{1}(p)
$$

where $\epsilon_{1}<0$. (Notice that any local one-dimensional attractive potential generates at least one bound state. The same is valid for the non-local potentials, $\left.V_{n n}\right)$. Using the spectral representation of the Greens function $G_{1}(E)$ we find for $E-E_{1} \rightarrow \epsilon_{1}$

$$
G_{1}(E)=\frac{\left|\tilde{\varphi}_{1}\right\rangle\left\langle\tilde{\varphi}_{1}\right|}{E-E_{1}-\epsilon_{1}}
$$

Then Eq. (2.24) is reduced to

$$
\left(E-E_{0}-\mathcal{K}_{0}-V_{00}\right) \psi_{0}=\hat{V} \psi_{0}
$$

where the potential $\hat{V}$ is the energy dependent and has the separable form

$$
\hat{V}\left(E, p, p^{\prime}\right)=\frac{\left\langle p\left|V_{01}\right| \tilde{\varphi}_{1}\right\rangle\left\langle\tilde{\varphi}_{1}\left|V_{10}\right| p^{\prime}\right\rangle}{E-E_{1}-\epsilon_{1}}
$$


Let us consider weak impurity potential, $\left|V_{00}\right| \ll E_{1}-E_{0}=\hbar \omega_{c}$. In this case one can neglect $V_{00}$ in Eqs. (2.22a), (3.3), which become

$$
\begin{gathered}
\left(E-E_{0}-\mathcal{K}_{0}\right) \psi_{0}=\hat{V} \psi_{0} \\
G_{0}\left(E ; p, p^{\prime}\right)=\frac{2 \pi \delta\left(p-p^{\prime}\right)}{E-E_{0}-\mathcal{K}_{0}(p)}
\end{gathered}
$$

However, the impurity potential in the r.h.s. of Eq. (3.5) cannot be neglected. It follows from Eq. (3.4) that $\hat{V} \rightarrow \infty$ when $E-E_{1} \rightarrow \epsilon_{1}$ for no matter how weak the impurity is. Therefore one cannot use any finite order Born approximation for Eq. (3.5), but rather the whole Born series

$$
\left|\psi_{0}\right\rangle=\left|k_{0}\right\rangle+G_{0} \hat{V}\left|k_{0}\right\rangle+G_{0} \hat{V} G_{0} \hat{V}\left|k_{0}\right\rangle+\cdots=\left(1+G_{0} t\right)\left|k_{0}\right\rangle
$$

where $\left\langle p \mid k_{0}\right\rangle=2 \pi \delta\left(p-k_{0}\right)$, and $t$ is the scattering operator, which satisfies the LippmannSchwinger equation

$$
t=\hat{V}+\hat{V} G_{0} t
$$

The wave vector $k_{0}$ is defined from the equation

$$
\mathcal{K}_{0}\left(k_{0}\right)=E-E_{0}
$$

Since $E-E_{0} \approx \hbar \omega_{c}$, one finds that $k_{0} \ell^{2} \sim a \gg \ell$ (see Fig. 1).

Substituting $\psi_{0}(p) \equiv\left\langle p \mid \psi_{0}\right\rangle$, given by Eq. (3.7) into Eq. (2.17) for $n=0$ and using Eq. (3.6) we get

$$
\tilde{\Psi}_{0, k_{0}}(p, y)=\left[2 \pi \delta\left(p-k_{0}\right)+\frac{\left\langle p|t| k_{0}\right\rangle}{E-E_{0}-\mathcal{K}_{0}(p)}\right] \tilde{\Phi}_{0, k_{0}}(p, y)
$$

The inverse Fourier transform $\tilde{\Psi}_{0, k_{0}}(p, y) \rightarrow \Psi_{0, k_{0}}(x, y)$ gives rise for the total wave function $\Psi_{0, k_{0}}(x, y)$ at $x \rightarrow+\infty$

$$
\Psi_{0, k_{0}}(x, y)=e^{i k_{0} x} \Phi_{0, k_{0}}(y)+\frac{1}{i \hbar v_{0}}\left\langle k_{0}|t| k_{0}\right\rangle e^{i k_{0} x} \Phi_{0, k_{0}}(y)
$$


where $v_{0}=\hbar^{-1}\left[d \mathcal{K}_{0}(p) / d p\right]_{p=k_{0}}$ is the carriers velocity inside the $n=0$ Landau mode. As a result, the corresponding transmission coefficient is

$$
T_{00}=\left|1+\frac{1}{i \hbar v_{0}}\left\langle k_{0}|t| k_{0}\right\rangle\right|^{2}
$$

where the scattering operator $t$ is given by the Lippmann-Schwinger equation (3.8). Since the potential $\hat{V}$ in Eq. (3.4) is a separable one, the Lippmann-Schwinger equation can be easily solved by taking $\left\langle p|t| p^{\prime}\right\rangle \equiv t\left(E, p, p^{\prime}\right)$ in a form

$$
t\left(E, p, p^{\prime}\right)=\frac{\left\langle p\left|V_{01}\right| \tilde{\varphi}_{1}\right\rangle\left\langle\tilde{\varphi}_{1}\left|V_{10}\right| p^{\prime}\right\rangle}{D(E)}
$$

Substituting Eq. (3.13) into Eq. (3.8) and using Eq. (3.4), we find that

$$
D(E)=E-E_{1}-\epsilon_{1}-\left\langle\tilde{\varphi}_{1}\left|V_{10} G_{0} V_{01}\right| \tilde{\varphi}_{1}\right\rangle
$$

Using Eq. (3.6) for $G_{0}$ one obtains

$$
\operatorname{Im}\left\langle\tilde{\varphi}_{1}\left|V_{10} G_{0} V_{01}\right| \tilde{\varphi}_{1}\right\rangle=-\left(\tilde{\Gamma}_{1}^{+}+\tilde{\Gamma}_{1}^{-}\right)
$$

where

$$
\tilde{\Gamma}_{1}^{ \pm}=\frac{1}{2 \hbar v_{0}}\left|\left\langle \pm k_{0}\left|V_{01}\right| \tilde{\varphi}_{1}\right\rangle\right|^{2}
$$

are the partial widths which acquires the bound state in the evanescent mode $n=1$ due to decay into the upper or into the lower edge states of the propagating mode $n=0$.

Substituting Eq. (3.13) into Eq. (3.12) and using Eqs. 3.14) - (3.16) we find

$$
T_{00}=\left|1-\frac{2 i \tilde{\Gamma}_{1}^{+}}{E-E_{1}-\epsilon_{1}-\tilde{\Delta}_{1}+i\left(\tilde{\Gamma}_{1}^{+}+\tilde{\Gamma}_{1}^{-}\right)}\right|^{2}
$$

where $\tilde{\Delta}_{1}=\operatorname{Re}\left\langle\tilde{\varphi}_{1}\left|V_{10} G_{0} V_{01}\right| \tilde{\varphi}_{1}\right\rangle$ is the shift of the bound state energy due to decay to the edge states (usually $\tilde{\Delta}_{1} \ll \epsilon_{1}$ ). Finally we obtain for the two-terminal conductance

$$
\mathcal{G}(E)=1-\frac{4 \tilde{\Gamma}_{1}^{+} \tilde{\Gamma}_{1}^{-}}{\left(E-\tilde{E}_{1}\right)^{2}+\left(\tilde{\Gamma}_{1}^{+}+\tilde{\Gamma}_{1}^{-}\right)^{2}} \equiv 1-\mathcal{R}(E)
$$

where $\tilde{E}_{1}=E_{1}+\epsilon_{1}+\tilde{\Delta}_{1}$, and 


$$
\mathcal{R}(E)=\left|t\left(E, k_{0},-k_{0}\right)\right|^{2} / \hbar^{2} v_{0}^{2}
$$

is the reflection coefficient. The latter is the probability of the inter-edge transition across a sample.

In general, when the electron energy approaches the threshold of the $N^{\prime}$ Landau mode, the same approach results in

$$
\mathcal{G}=N-\frac{4 \tilde{\Gamma}_{N}^{+} \tilde{\Gamma}_{N}^{-}}{\left(E-\tilde{E}_{N}\right)^{2}+\left(\tilde{\Gamma}_{N}^{+}+\tilde{\Gamma}_{N}^{-}\right)^{2}}
$$

where the expressions for $\tilde{\Gamma}_{N}^{ \pm}$and $\tilde{E}_{N}$ are completely analogous to those for $N=1$. Similar result for the total conductance $\mathcal{G}$ in a case of short range impurity has been obtained in Ref. [8] by using a different technique.

We thus demonstrated that any weak attractive impurity generates the resonance dip in the total conductance (the peak in the longitudinal resistance). This dip (peak) appears as a result of the backward scattering due to inter-edge transition via the resonance state in the nearest evanescent mode. This inter-mode scattering mechanism is typical for an attractive impurity which produces bound states below thresholds of the Landau modes.

\section{B. Attractive short-range impurity}

Now we apply the results obtained above for the evaluation of the resonance energy and the partial widths in a case of an attractive short range impurity potential, i.e. the interaction range is much smaller than the magnetic length $\ell$. In this case one can write for $V_{n n^{\prime}}$, Eq. (2.19),

$$
\begin{aligned}
V_{n n^{\prime}}\left(p, p^{\prime}\right) & =\int \tilde{\Phi}_{n^{\prime}}^{*}\left(p^{\prime}, y\right) V\left(x-x_{i}, y-y_{i}\right) \tilde{\Phi}_{n}(p, y) e^{-i\left(p-p^{\prime}\right) x} d x d y \\
& \simeq \lambda \Phi_{n}\left(y_{i}-\ell^{2} p\right) \Phi_{n^{\prime}}\left(y_{i}-\ell^{2} p^{\prime}\right)
\end{aligned}
$$

where $\lambda=\int V(x, y) d x d y<0$ and $\Phi_{n}$ are the Harmonic oscillator wave functions, Eq. (2.5). The impurity coordinate $x_{i}$ is chosen zero. Here we assumed that that the impurity is not 
in the edge regions, so that the Landau-mode wave functions can be well approximated by the harmonic oscillator functions, $\tilde{\Phi}_{n}(p, y)=\Phi_{n}\left(y_{i}-\ell^{2} p\right)$.

Since the potential $V_{11}$, Eq. (3.21) has a separable form, the Schroedinger equation (3.1) for the bound state can be easily solved. One obtains

$$
\tilde{\varphi}_{1}(p)=\mathcal{N} \frac{\Phi_{1}\left(y_{i}-\ell^{2} p\right)}{\epsilon_{1}-\mathcal{K}_{1}(p)}
$$

where $\mathcal{N}$ is the normalization:

$$
\mathcal{N}^{-2}=\int \frac{\Phi_{1}^{2}\left(y_{i}-\ell^{2} p\right)}{\left[\epsilon_{1}-\mathcal{K}_{1}(p)\right]^{2}} \frac{d p}{2 \pi}
$$

and the bound state energy $\epsilon_{1}$ is defined by the equation

$$
1-\lambda \int \frac{\Phi_{1}^{2}\left(y_{i}-\ell^{2} p\right)}{\epsilon_{1}-\mathcal{K}_{1}(p)} \frac{d p}{2 \pi}=0
$$

Since $\mathcal{K}_{1}(p) \geq 0$, it is clear that for the attractive interaction $(\lambda<0)$, and no matter how small the $\lambda$ is, there always exists $\epsilon_{1}<0$, which satisfies Eq. (3.24). One can easily evaluate $\epsilon_{1}$ and $\mathcal{N}$ by taken into account that $\mathcal{K}_{1} \cong 0$ in the region of $p$ which makes the dominant contribution in the integrals (3.23), (3.24). Then

$$
\epsilon_{1}=\frac{\lambda}{2 \pi \ell^{2}}, \quad \mathcal{N}=\left(\lambda \epsilon_{1}\right)^{1 / 2}
$$

The bound state $\tilde{\varphi}_{1}$ which appears in the evanescent mode $(n=1)$, generates the resonance in the propagating mode $(n=0)$ through the mode-mixing potential $V_{01}$, Eq. (3.21)

$$
V_{01}\left(p, p^{\prime}\right)=\lambda \Phi_{0}\left(y_{i}-\ell^{2} p\right) \Phi_{1}\left(y_{i}-\ell^{2} p^{\prime}\right)
$$

Substituting Eq. (3.26) into Eq. (3.16) and using Eqs. (2.5) and (3.25) we find for the partial widths

$$
\tilde{\Gamma}_{1}^{ \pm}=\frac{\epsilon_{1}^{2} \sqrt{\pi} \ell}{\hbar \tilde{v}_{0}} \exp \left[-\left(y_{i} \mp \ell^{2} \tilde{k}_{0}\right)^{2} / \ell^{2}\right]
$$

where $\tilde{k}_{0}$ is the electron wave vector at the resonance energy, which is defined from the equation $\mathcal{K}_{0}\left(\tilde{k}_{0}\right)=E_{1}-E_{0}-\epsilon_{1}$, and the velocity $\tilde{v}_{0}=\hbar^{-1}\left[d \mathcal{K}_{0}(p) / d p\right]_{p=\tilde{k}_{0}}$. One can estimate from Fig. $1 \mathrm{~b}$ that $\tilde{k}_{0} \ell^{2} \sim a$ and $\tilde{v}_{0} \sim \ell \omega_{c}$. 
It follows from Eq. (3.27) that $\tilde{\Gamma}_{1}^{+} / \tilde{\Gamma}_{1}^{-} \sim \exp \left(4 y_{i} a / \ell^{2}\right)$. Therefore for a wide sample $(a / \ell \gg 1)$ the resonance partial widths $\tilde{\Gamma}_{1}^{+}$and $\tilde{\Gamma}_{1}^{-}$are largely different unless $4 y_{i} a \sim \ell^{2}$. As a result the depth of the resonant dip in the total conductance, Eq. (3.18), is exponentially small

$$
(\Delta \mathcal{G})_{\max }=\mathcal{R}\left(\tilde{E}_{1}\right)=\frac{4 \tilde{\Gamma}_{1}^{+} / \tilde{\Gamma}_{1}^{-}}{\left(\tilde{\Gamma}_{1}^{+} / \tilde{\Gamma}_{1}^{-}+1\right)^{2}} \sim 4 e^{-4 \tilde{k}_{0} y_{i}}
$$

and therefore the resonance is strongly suppressed, unless the impurity is not in the middle of a sample [8]. Then the resonance peak appears in the longitudinal resistance, although its width would be exponentially small.

\section{Short range repulsive impurity}

Using classical mechanics arguments one finds that a repulsive impurity potential in the presence of strong magnetic field can trap electrons moving in a confining conducting channel. However, in quantum mechanical sense, such a state is not a bound one. It is rather a quasi-bound (resonance) state because the energy spectrum of the corresponding Hamiltonian, Eq. (2.1), is continuous and there are no normalized eigenstates exist in continuum spectrum. Similar to the previous case such a quasi-bound state can generate resonant transitions of carriers between the edges. The difference is that the resonances from repulsive impurities appear above thresholds of the propagating Landau modes, whereas the resonances generated by attractive impurities are below thresholds. As a result repulsive impurities can produce direct inter-edge transitions inside the propagating modes (the inner-mode transitions), in a contrast with repulsive impurities, which generate inter-edge transitions via bound states in the evanescent modes, (the inter-mode transitions).

Let us investigate these resonances using the same couple-channel technique. Consider the electron energy $E$ in vicinity of the threshold of the $n=0$ Landau mode, $E \sim E_{0}$. If the impurity is weak, $V_{01} G_{1}(E) V_{01} \sim\left|V_{00}\right|^{2} /\left(E_{1}-E_{0}\right) \ll V_{00}$, the right-hand side term in Eq. (2.24) can be neglected. Then this equation can be rewritten as 


$$
\left(E-E_{0}-\mathcal{K}_{0}-V_{00}\right) \psi_{0}=0
$$

Notice that $V_{00}$ is a non-local one-dimensional repulsive potential. Therefore it can generate resonances, though a local one-dimensional repulsive potential cannot.

We start with a short range repulsive impurity. Then the potential $V_{00}$, Eq. (3.21), has the same separable form as in the case of an attractive short range impurity:

$$
V_{00}\left(p, p^{\prime}\right)=\lambda g_{0}(p) g_{0}\left(p^{\prime}\right)
$$

where $\lambda=\int V(x, y) d x d y>0$ and $g_{0}(p)=\Phi_{0}\left(y_{i}-\ell^{2} p\right)$. The corresponding transmission coefficient is given by Eq. (3.12), with the scattering operator $t$ obtained from the LippmannSchwinger equation

$$
t=V_{00}\left(1+G_{0} t\right)
$$

and the reflection coefficient, $\mathcal{R}(E)$, i.e. the probability of the inter-edge transition, is given by Eq. (3.19). Taking $t\left(E, p, p^{\prime}\right)$ in a form

$$
t\left(E, p, p^{\prime}\right)=\lambda g_{0}(p) g_{0}\left(p^{\prime}\right) / D(E)
$$

we get

$$
D(E)=1-\int \frac{\lambda g_{0}^{2}\left(p^{\prime \prime}\right)}{E-E_{0}-\mathcal{K}_{0}\left(p^{\prime \prime}\right)+i \delta} \frac{d p^{\prime \prime}}{2 \pi}
$$

Since $\lambda$ is positive, one easily finds that $\operatorname{Re}[D(E)]$ vanishes for $E=E_{0}+\epsilon_{0}$, where $\epsilon_{0}>0$ is defined from the equation

$$
1-\mathcal{P} \int \frac{\lambda g_{0}^{2}\left(p^{\prime \prime}\right)}{\epsilon_{0}-\mathcal{K}_{0}\left(p^{\prime \prime}\right)} \frac{d p^{\prime \prime}}{2 \pi}=0
$$

Here $\mathcal{P}$ denotes the principal value of the integral. Hence, one gets for $E-E_{0} \sim \epsilon_{0}$,

$$
D(E)=\mathcal{A}\left[E-E_{0}-\epsilon_{0}+i\left(\Gamma_{0}^{+}+\Gamma_{0}^{-}\right)\right]
$$

where $\mathcal{A}=d[\operatorname{Re} D(E)] /\left.d E\right|_{E=E_{0}+\epsilon_{0}}$ and $\Gamma_{0}^{ \pm}$are obtained from the singular part of Eq.(3.33). As in the previous case of attractive impurity one can evaluate the resonance energy $\epsilon_{0}$ and $\mathcal{A}$ by taken $\mathcal{K}_{0} \sim 0$ in Eq. (3.34), thus obtaining 


$$
\epsilon_{0}=\lambda \int g_{0}^{2}\left(p^{\prime \prime}\right) \frac{d p^{\prime \prime}}{2 \pi}=\frac{\lambda}{2 \pi \ell^{2}}
$$

and $\mathcal{A}=\epsilon_{0}^{-1}$, where the partial widths are given by

$$
\Gamma_{0}^{ \pm}=\frac{\lambda}{2 \bar{v}_{0}} \mathcal{A}^{-1} g_{0}^{2}\left( \pm \bar{k}_{0}\right)=\frac{\epsilon_{0}^{2} \sqrt{\pi} \ell}{\hbar \bar{v}_{0}} \exp \left[-\left(y_{i} \mp \ell^{2} \bar{k}_{0}\right)^{2} / \ell^{2}\right]
$$

Here $\bar{k}_{0}$ is determined from the equation $\mathcal{K}_{0}\left(\bar{k}_{0}\right)=\epsilon_{0}$, and $\bar{v}_{0}=\hbar^{-1}\left[d \mathcal{K}_{0}(p) / d p\right]_{p=\bar{k}_{0}}$ is the carrier velocity.

Substituting Eq. (3.35) into Eq. (3.32) we find that the scattering amplitude $t\left(E, p, p^{\prime}\right)$ has the Breit-Wigner form, which means the existence of the resonance above the Hall plateau at the energy $\bar{E}_{0}=E_{0}+\epsilon_{0}$ and of the width $\Gamma_{0}^{+}+\Gamma_{0}^{-}$. Using Eq. (3.12) we finally obtain for the two-terminal conductance $\mathcal{G}$ in vicinity of the resonance: $\mathcal{G}=1-\mathcal{R}$, where

$$
\mathcal{R}(E)=\frac{4 \Gamma_{0}^{+} \Gamma_{0}^{-}}{\left(E-\bar{E}_{0}\right)^{2}+\left(\Gamma_{0}^{+}+\Gamma_{0}^{-}\right)^{2}}
$$

We thus found that both repulsive and attractive impurities produce the resonance dip in the total conductance (and therefore the peak in the longitudinal resistance). It both cases the resonance energy and the width are given by the same expressions, Eqs. (3.37),(3.36), and Eqs. 3.25), (3.27), respectively, though the resonances appear near different Landau modes. Notice that the carrier velocity $\bar{v}_{0}$ in Eq. (3.37) is smaller than the corresponding velocity $\tilde{v}_{0}$ entering Eq. (3.27) (see Fig. 1a). As a result the resonance generated by a single repulsive impurity is more broadened than that from a single attractive impurity. However, the mostly pronounced distinction between these two cases takes place for extremely weak impurities, $\lambda \rightarrow 0$. Then the values of the corresponding wave vectors, $\tilde{k}_{0}$ and $\bar{k}_{0}$, are very different. The wave vector $\tilde{k}_{0}$ for the attractive impurity is always large, $\tilde{k}_{0} \ell \sim a / \ell \gg 1$, even when $\lambda \rightarrow 0$ (see Fig. 1a). In a contrast, the wave vector $\bar{k}_{0} \rightarrow 0$ for $\lambda \rightarrow 0$. Indeed, one obtains from Eq. (2.6) for $\epsilon_{0} / \hbar \omega_{c} \sim \exp \left(-\bar{a}^{2}\right)$

$$
\bar{k}_{0} \ell=\left(\frac{\sqrt{\pi}}{4 \bar{a}^{3}} e^{\bar{a}^{2}} \frac{\epsilon_{0}}{\hbar \omega_{c}}\right)^{1 / 2}=\left(\frac{\lambda \ell}{8 a^{3} \sqrt{\pi} \hbar \omega_{c}}\right)^{1 / 2} e^{a^{2} / 2 \ell^{2}}
$$

Correspondingly, the partial widths, Eq. (3.37), are 


$$
\Gamma_{0}^{ \pm}=\frac{\epsilon_{0}^{3 / 2}}{\left(\hbar \omega_{c}\right)^{1 / 2}} \frac{\pi^{3 / 4}}{4 \bar{a}^{3 / 2}} e^{\bar{a}^{2} / 2} \exp \left[-\left(\frac{y_{i}}{\ell} \mp \bar{k}_{0} \ell\right)^{2}\right]
$$

Therefore, $\Gamma_{0}^{+} \sim \Gamma_{0}^{-}$, irrespectively on the position of the impurity $y_{i}$, since $\bar{k}_{0} \ell \ll 1$. As a result the resonance dip of total conductance, Eq. (3.38), may survive even in a wide sample. Such a different behavior of partial widths for attractive and repulsive impurities is easy to interpret by notice that the very weak attractive impurity generates the resonance just above the Hall plateau, where the corresponding wave vector is small. In this case the effective electron spreading is large and therefore the resonant scattering would be insensitive to the impurity position.

\section{General case}

If the repulsive impurity potential, $V(x, y)$, is not a short range one, then the corresponding non-local potential $V_{00}$ in Eq. (3.29) cannot be written in the separable form of Eq. (3.30). In this case our previous treatment cannot be applied. However, one can benefit by the effective kinetic term $\mathcal{K}_{0}(p)$ is almost zero except for $p \sim \pm a / \ell^{2}$. That allows to consider the kinetic term as a perturbation. Let us put $\mathcal{K}_{0}=0$ in Eq. (3.29) (the infinite mass approximation). In this case the resonance state $\psi_{0}(p)$, generated by Eq. (3.29) appears as a stable state described by the normalized wave function, $\psi_{0}(p) \rightarrow \varphi_{0}(p)$, and Eq. (3.29) can be rewritten as

$$
\int V_{00}\left(p, p^{\prime}\right) \varphi_{0}\left(p^{\prime}\right) \frac{d p^{\prime}}{2 \pi}=\epsilon_{0} \varphi_{0}(p)
$$

Here $\epsilon_{0}=E-E_{0}$ is the resonance energy.

When the neglected kinetic term $\mathcal{K}_{0}$ is "turned on", the bound state $\varphi_{0}$ gets the width, because $\mathcal{K}_{0}$ provides the coupling with continuum of the edge states. In order to calculate the partial widths we apply the approach developed in [11,12] for treatment of the resonance states. We found in [1] that the corresponding partial widths are obtained in terms of the matrix elements of the impurity potential between the bound state and the free (continuum) states 


$$
\Gamma_{0}^{ \pm}=\frac{1}{2 \hbar \bar{v}_{0}}\left|<\varphi_{0}\right| V_{00}\left|\mp \bar{k}_{0}>\right|^{2}=\frac{\epsilon_{0}^{2}}{2 \hbar \bar{v}_{0}}\left|<\varphi_{0}\right| \mp \bar{k}_{0}>\left.\right|^{2}
$$

where Eq. (3.41) has been used in order to eliminate the impurity potential $V_{00}$. Correspondingly, the scattering amplitude $t$, Eq. (3.31), in the vicinity of the resonance is given by the Breit-Wigner formula

$$
t\left(E, p, p^{\prime}\right)=\frac{\epsilon_{0}^{2}<p\left|\varphi_{0}><\varphi_{0}\right| p^{\prime}>}{E-E_{0}-\epsilon_{0}+i\left(\Gamma_{0}^{+}+\Gamma_{0}^{-}\right)}
$$

Finally, the probability of the inter-edge transition (the reflection coefficient) is determined by Eq. (3.19), namely $\mathcal{R}=\left|t\left(E, \bar{k}_{0},-\bar{k}_{0}\right)\right|^{2} / \hbar^{2} \bar{v}_{0}^{2}$.

As an example, let us apply Eqs. (3.41), (3.42) for a short range repulsive potential. Substituting Eq. (3.30) into Eq. (3.41) one finds that the solution is

$$
\varphi_{0}(p)=C g_{0}(p)
$$

where $C=(2 \pi \ell)^{1 / 2}$ is the normalization coefficient, and the resonance energy $\epsilon_{0}$ is given by Eq. (3.36). Also, substituting Eq. (3.44) into Eq. (3.42) we reproduce Eq. (3.37) for the partial widths. Notice that Eq. (3.41) has no solutions if $V_{00}$ is a local potential: $V_{00} \equiv V_{00}\left(p-p^{\prime}\right)$.

\section{E. Repulsive impurity of Gaussian-type}

Let us consider an another example of the carrier-impurity interaction, namely the Gaussian potential

$$
V(x, y)=\frac{\lambda}{\pi r_{0}^{2}} \exp \left[-\left(\boldsymbol{r}-\boldsymbol{r}_{i}\right)^{2} / r_{0}^{2}\right]
$$

where $\boldsymbol{r}=(x, y)$, and $\boldsymbol{r}_{i}=\left(x_{i}, y_{i}\right)$. The interaction range $r_{0}$ is taken as a free parameter. Hence, $V(x, y)$ can describe either short or long-range impurity. We restrict ourself with a repulsive interaction $(\lambda>0)$, although our treatment can be easily performed for an attractive interaction as well. 
Using Eqs. (2.5), (2.19) and taking $x_{i}=0$ we get for the potential $V_{00}$ the following expression:

$$
V_{00}\left(p, p^{\prime}\right)=\frac{\lambda}{\sqrt{\pi} R_{0}} \exp \left[-\frac{\ell^{2}}{R_{0}^{2}}\left(\frac{y_{i}}{\ell}-\ell \frac{p+p^{\prime}}{2}\right)^{2}-\frac{R_{0}^{2}}{4}\left(p-p^{\prime}\right)^{2}\right]
$$

where $R_{0}=\left(r_{0}^{2}+\ell^{2}\right)^{1 / 2}$. Notice that for $r_{0} \ll \ell$ (the short range interaction)

$$
V_{00}\left(p, p^{\prime}\right) \rightarrow \frac{\lambda}{\sqrt{\pi} \ell} e^{-\left(y_{i}-\ell^{2} p\right)^{2} / 2 \ell^{2}} e^{-\left(y_{i}-\ell^{2} p^{\prime}\right)^{2} / 2 \ell^{2}}
$$

i.e. the potential obtains the separable form of Eq.(3.30). However, in a case of long range interaction, $r_{0} \gg \ell$, the potential turns to a local one:

$$
V_{00}\left(p, p^{\prime}\right) \rightarrow \frac{\lambda}{\sqrt{\pi} r_{0}} e^{-\left[4 y_{i}^{2}+r_{0}^{4}\left(p-p^{\prime}\right)^{2}\right] / 4 r_{0}^{2}}
$$

Substituting Eq. (3.46) into Eq. (3.41) and using the variables $q=y_{i}-\ell^{2} p$ and $q^{\prime}=$ $y_{i}-\ell^{2} p^{\prime}$ instead of $p$ and $p^{\prime}$ we get

$$
\int \tilde{V}_{00}\left(q, q^{\prime}\right) \varphi_{0}\left(q^{\prime}\right) \frac{d q^{\prime}}{2 \pi \ell^{2}}=\epsilon_{0} \varphi_{0}(q)
$$

where

$$
\tilde{V}_{00}\left(q, q^{\prime}\right)=\frac{\lambda}{\sqrt{\pi} R_{0}} e^{-\alpha\left(q^{2}+q^{2}\right)+2 \beta q q^{\prime}}
$$

Here the parameters $\alpha$ and $\beta$ are

$$
\alpha=\frac{R_{0}^{4}+\ell^{4}}{4 R_{0}^{2} \ell^{4}}, \quad \beta=\frac{R_{0}^{4}-\ell_{0}^{4}}{4 R_{0}^{2} \ell^{4}}
$$

One finds that the wave function $\varphi_{0}$ taken in a Gaussian form

$$
\varphi_{0}(q)=2^{3 / 4}(\pi \eta)^{1 / 4} \ell \exp \left(-\eta q^{2}\right)
$$

satisfies Eq. (3.49) for $\eta=1 / 2 \ell^{2}$, where the resonance energy is

$$
\epsilon_{0}=\frac{\lambda}{\pi\left(R_{0}^{2}+\ell^{2}\right)}
$$

Using Eqs. (3.42) and (3.52) we obtain for the partial widths the following result 


$$
\Gamma_{0}^{ \pm}=\frac{\epsilon_{0}^{2} \sqrt{\pi} \ell}{\hbar \bar{v}_{0}} \exp \left[-\left(y_{i} \mp \ell^{2} \bar{k}_{0}\right)^{2} / \ell^{2}\right]
$$

which coincides with Eq. (3.37) for a short range impurity.

Comparing $\varphi_{0}(q)$ given by Eq. (3.52) with the wave function for a short range impurity, Eq. (3.44), we find that the both wave functions coincide. It is rather remarkable that the range of the Gaussian potential does not enter into $\varphi_{0}$, so that the wave function spreading is the same as for a short range impurity. The interaction range $r_{0}$ appears only in the resonance energy, Eq. (3.53). One finds that in the limit of $r_{0} \rightarrow \infty$ the energy $\epsilon_{0} \rightarrow 0$, and the resonance disappears. This result is rather expectable since the potential $V_{00}$ in this limit turns to be a local repulsive potential, Eq. (3.48), which does not produces any quasi-stationary states.

\section{RESONANT SCATTERING ON TWO IMPURITIES}

It was found in the previous section that the inter-edge resonant transport via single impurity drops down very rapidly with a sample width. Let us consider an another mechanism of inter-edge transport, when a carrier reaches the opposite edge through subsequent resonant hopping via many impurities. We start with an example of a such resonant hopping via two identical impurities. In this case the potential $V(x, y)$ in Eq. (2.1) is

$$
V(x, y)=V\left(x-x_{1}, y-y_{1}\right)+V\left(x-x_{2}, y-y_{2}\right)
$$

For the definiteness we discuss two repulsive impurities, although the analysis is valid for attractive impurities as well.

Consider Eq. (3.29) near the $n=0$ Landau mode threshold. Using Eq. (2.19) one can rewrite the potential $V_{00}$ as

$$
V_{00}\left(p, p^{\prime}\right)=\bar{V}_{00}^{(1)}\left(p, p^{\prime}\right) e^{-i\left(p-p^{\prime}\right) x_{1}}+\bar{V}_{00}^{(2)}\left(p, p^{\prime}\right) e^{-i\left(p-p^{\prime}\right) x_{2}}
$$

It follows from our previous analysis that each of impurity potentials in Eq. (4.2) generates the resonance above the Hall plateau at the same energy, $\bar{E}_{0}=E_{0}+\epsilon_{0}$, where $\epsilon_{0}=\lambda / 2 \pi \ell^{2}$, 
Eq. (3.36). Neglecting the kinetic term $\mathcal{K}_{0}$ in Eq. (3.29), one finds that each of the resonances becomes stable state, described by the normalized wave functions

$$
\varphi_{j}(p)=\bar{\varphi}_{j}(p) \exp \left(-i p x_{j}\right)
$$

where $j=1,2$ and $\bar{\varphi}_{j}(p)$ is obtained from Eq. (3.41) for $V_{00}\left(p, p^{\prime}\right)=\bar{V}_{00}^{j}\left(p, p^{\prime}\right)$. Notice that the phase factor in Eq. (4.3) would play now an important role, although it was insignificant in the single scattering process. Similar to the previous case, the states $\varphi_{j}(p)$ obtain the widths $\Gamma_{j}=\Gamma_{j}^{+}+\Gamma_{j}^{-}$, when the neglected kinetic term $\mathcal{K}_{0}$ is "switched on". Here $\Gamma_{j}^{ \pm}$denote the partial widths due to decay into the upper and the lower edge state respectively, Eq. (3.37).

One can easily realize that the resonance transport of carriers via two impurities is completely analogous to the resonant tunneling through one-dimensional double-well potential system with aligned levels. This process has been studied in details by using different techniques, see for instance [13 [15] and references therein. Here we adopt the time-dependent approach of Ref. [15], since it is easily extended for a case of many impurities. According to this approach the amplitude of resonant scattering on two impurities can be obtained directly from the Schroedinger equation $i \hbar \dot{\psi}(t)=\mathcal{H} \psi(t)$ for two states wave function $\psi(t)=\left(b_{1}(t), b_{2}(t)\right)$, with the initial condition $\psi(0)=(1,0)$, where $b_{1,2}(t)$ are the probability amplitudes to find the carrier in the states $\varphi_{1,2}$ respectively, and $\mathcal{H}$ is the effective Hamiltonian

$$
\mathcal{H}=\left(\begin{array}{cc}
\bar{E}_{0}-i \Gamma_{1} & \Omega_{21} \\
\Omega_{12} & \bar{E}_{0}-i \Gamma_{2}
\end{array}\right)
$$

The off-diagonal matrix element $\Omega_{12}$ is the hopping transition amplitude between the states, $\varphi_{1,2}$. The diagonal energy shifts, $\Delta_{1}=\Delta_{2} \ll\left|\Omega_{12}\right|$ (see Appendix B) were included in the energy $\bar{E}_{0}$. The amplitude $b_{2}$ determines the scattering amplitude Eq. (3.31) in a vicinity of the resonance, $E \sim \bar{E}_{0}=E_{0}+\epsilon_{0}$ by means of

$$
\hbar t\left(E, p, p^{\prime}\right)=<p\left|V_{00}^{(1)}\right| \varphi_{1}>\tilde{b}_{2}(E)<\varphi_{2}\left|V_{00}^{(2)}\right| p^{\prime}>=\epsilon_{0}^{2}<p\left|\varphi_{1}>\tilde{b}_{2}(E)<\varphi_{2}\right| p^{\prime}>
$$

(c.f. with Eq. (3.43)), where $\tilde{b}_{j}$ is the Laplace transform of $b_{j}$ : 


$$
\tilde{b}_{j}(E)=\int_{0}^{\infty} \exp (i E t / \hbar) b_{j}(t) d t
$$

Solving these equations we obtain for the probability of the inter-edge transition, $\mathcal{R}(E)$, Eq. (3.19), the following result

$$
\mathcal{R}(E)=\frac{4 \Gamma_{1}^{+}\left|\Omega_{12}\right|^{2} \Gamma_{2}^{-}}{|\operatorname{det}[E-\mathcal{H}]|^{2}}
$$

where

$$
\operatorname{det}[E-\mathcal{H}]=\left(E-\bar{E}_{0}+i \Gamma_{1}\right)\left(E-\bar{E}_{0}+i \Gamma_{2}\right)-\left|\Omega_{12}\right|^{2}
$$

Thus, $\mathcal{R}(E)$ coincides with the probability of the resonance passage through a double-well potential for aligned levels [13,14]. One finds from Eqs. (4.7), (4.8) that $\mathcal{R}(E)$ has two peaks near the Landau levels at the energies

$$
E_{ \pm}=\bar{E}_{0} \pm \sqrt{\left|\Omega_{12}\right|^{2}-\frac{\left(\Gamma_{1}-\Gamma_{2}\right)^{2}}{4}}
$$

which reflects the splitting of the resonance due to off-diagonal transitions between the two impurities. The reflection coefficient $\mathcal{R}(E)$ reaches the maxima at $E=E_{ \pm}$

$$
\mathcal{R}_{\max }=\frac{4 \Gamma_{1}^{+} \Gamma_{2}^{-}}{\left(\Gamma_{1}^{+}+\Gamma_{2}^{-}\right)^{2}} \frac{\left|\Omega_{12}\right|^{2}}{\left|\Omega_{12}\right|^{2}+\frac{\left(\Gamma_{1}^{+}+\Gamma_{2}^{-}\right)^{2}}{16}}
$$

It is quite clear from Eq. (4.10) that the inter-edge transition via two impurities is more likely than the direct transition via one impurity. For instance, it is not required for the impurities to be in the middle of a sample in order to generate non-vanishing resonance peak in $\mathcal{R}(E)$.

Using Eq. (B11) from Appendix B for $\mathcal{K}_{0}=0$, and Eqs. (3.44) for the wave functions $\varphi_{1,2}$, in a case of short range (or Gaussian) impurity potentials, we obtain for the hopping matrix element

$$
\Omega_{i j}=\epsilon_{0} \int \varphi_{i}(p) \varphi_{j}^{*}(p) \frac{d p}{2 \pi}=\epsilon_{0} e^{-\left(\boldsymbol{r}_{i}-\boldsymbol{r}_{j}\right)^{2} / 4 \ell^{2}} e^{-i\left(x_{i}-x_{j}\right)\left(y_{i}+y_{j}\right) / 2 \ell^{2}}
$$

where for the diagonal energy shifts $\Delta_{1}=\Delta_{2}$ one gets (for a short range potential) 


$$
\Delta_{i}=\int \varphi_{i}(p) V_{00}^{j}\left(p, p^{\prime}\right) \varphi_{i}^{*}\left(p^{\prime}\right) \frac{d p d p^{\prime}}{4 \pi^{2}}=\epsilon_{0} e^{-\left(\boldsymbol{r}_{i}-\boldsymbol{r}_{j}\right)^{2} / 2 \ell^{2}}
$$

As expected, $\Delta_{i} \ll\left|\Omega_{i j}\right|$.

It is important to point out that the non-diagonal hopping elements are complex, $\Omega_{i j} \equiv$ $\left|\Omega_{i j}\right| \exp \left(-i \alpha_{i j}\right)$, with the phase

$$
\alpha_{i j}=\pi B\left(x_{i}-x_{j}\right)\left(y_{i}+y_{j}\right) / \phi_{0}
$$

where $\phi_{0}=h c / e$ is the flux quantum.

\section{RESONANT SCATTERING ON $N$ IMPURITIES}

\section{A. Interference effects}

Our results for resonant scattering on two impurities can be extended for any number of impurities inside a sample. Let us consider the transport of a carrier from the upper to the lower edge as a sequence of resonant transitions via $n$ impurities located at the points $\boldsymbol{r}_{1}, \ldots, \boldsymbol{r}_{n}$ inside a sample. We enumerate impurities in such a way that the first one is closest to the upper edge, and the last one is closest to the lower edge. As in the previous case we describe this process by the $n$-states wave function $\psi(t)=\left(b_{1}(t), \ldots, b_{n}(t)\right)$, with $b_{i}(t)$ is the

amplitude for the carrier to be in the state $\varphi_{j}$ on the $i$-impurity site, which is a solution of the Schroedinger equation $i \hbar \dot{\psi}(t)=\mathcal{H} \psi(t)$ for the initial condition $\psi(0)=(1,0, \ldots, 0)$, and the effective Hamiltonian is

$$
\mathcal{H}=\left(\begin{array}{ccccc}
\bar{E}_{0}-i \Gamma_{1} & \Omega_{21} & \Omega_{31} & \cdots & \Omega_{n 1} \\
\Omega_{12} & \bar{E}_{0} & \Omega_{32} & \cdots & \Omega_{n 2} \\
\Omega_{13} & \Omega_{23} & \bar{E}_{0} & \cdots & \Omega_{n 3} \\
\cdots & \cdots & \cdots & \cdots & \cdots \\
\Omega_{1 n} & \Omega_{2 n} & \Omega_{3 n} & \cdots & \bar{E}_{0}-i \Gamma_{n}
\end{array}\right)
$$

where $\bar{E}_{0}=E_{0}+\epsilon_{0}$. Here the coupling with the continuum of the edge states (through the width $\Gamma$ ) is kept only for the first and for the last impurities, since all the others are 
farther away from the edges. The widths $\Gamma_{1, n}$ and the hopping amplitudes $\Omega_{i j}$ are given by Eq. (3.37) and Eq. (4.11) respectively. We neglected the diagonal energy shift $\Delta_{i}$ in the Hamiltonian, since $\Delta_{i} \ll|\Omega|$, Eq. (4.12), although its average value can be included in $\bar{E}_{0}$. The amplitude $\tilde{b}_{n}(E)$, which is the Laplace transform of $b_{n}(t)$, Eq. (4.6), defines the probability of the inter-edge transition

$$
\mathcal{R}(E)=4 \Gamma_{1}\left|\tilde{b}_{n}(E)\right|^{2} \Gamma_{n}
$$

Notice that the described procedure is very similar to a treatment of resonant tunneling in multi-well heterostructures [16].

It is important to point out that each impurity generates the quasi-bound state at the same energy $\bar{E}_{0}=E_{0}+\epsilon_{0}$, irrespectively on the impurity position. As a result, all the diagonal elements in the Hamiltonian (5.1) are the same, except for the first and the last one, which obtain the imaginary part due to the coupling with the edge states. However, the off-diagonal matrix elements $\Omega_{i j}$ are widely different. Since $\Omega_{i j}$ drops down very fast for $\left|\boldsymbol{r}_{i}-\boldsymbol{r}_{j}\right|>2 \ell$, one can expect to find only a few paths of a carrier via the $n$-impurities which would contribute to the inter-edge transport . Hence, it is not necessary to keep all the amplitudes $\Omega_{i j}$ in the Hamiltonian (5.1), but only those which are associated with the most probable path of the resonant transitions.

If two or more most probable trajectories connect the impurities 1 and $n$ (self-crossing trajectories), an additional Aharonov-Bohm oscillatory factor would appear in $\mathcal{R}(E)$. We now demonstrate on a simple example how our treatment reproduces this interference effect. Let us consider four impurities in the channel for a configuration shown in Fig. 3. We assume that $\left|\boldsymbol{r}_{1}-\boldsymbol{r}_{2}\right|=\left|\boldsymbol{r}_{1}-\boldsymbol{r}_{3}\right|$ and $\left|\boldsymbol{r}_{2}-\boldsymbol{r}_{4}\right|=\left|\boldsymbol{r}_{3}-\boldsymbol{r}_{4}\right|$. Therefore $\left|\Omega_{12}\right|=\left|\Omega_{13}\right|$ and $\left|\Omega_{24}\right|=\left|\Omega_{34}\right|$. Since $\left|\Omega_{14}\right| \ll\left|\Omega_{12}\right|,\left|\Omega_{23}\right|,\left|\Omega_{24}\right|$ we neglect the direct transition between the first and the last impurities by putting $\Omega_{14}=0$. Then the effective Hamiltonian (5.1) can be written as 


$$
\mathcal{H}=\left(\begin{array}{cccc}
\bar{E}_{0}-i \Gamma_{1} & \Omega_{21} & \Omega_{31} & 0 \\
\Omega_{12} & \bar{E}_{0} & \Omega_{32} & \Omega_{42} \\
\Omega_{13} & \Omega_{23} & \bar{E}_{0} & \Omega_{43} \\
0 & \Omega_{24} & \Omega_{34} & \bar{E}_{0}-i \Gamma_{4}
\end{array}\right)
$$

The probability of the inter-edge transition, $\mathcal{R}(E)$, is given by Eq. (5.2) for $n=4$, where the

4-state wave function $\tilde{\psi}(E)=\left(\tilde{b}_{1}(E), \tilde{b}_{2}(E), \tilde{b}_{3}(E), \tilde{b}_{4}(E)\right)$ is obtained from the Schroedinger equation in the Laplace-transform form

$$
\sum_{j^{\prime}}\left(E \delta_{j j^{\prime}}-\mathcal{H}_{j j^{\prime}}\right) \tilde{b}_{j^{\prime}}(E)=i \hbar \delta_{1 j}
$$

where the r.h.s. reflects the initial condition $\psi(t=0)=(1,0,0,0)$. By solving Eq. (5.4) we find

$$
\tilde{b}_{4}(E)=i \hbar \frac{\left(\Omega_{12} \Omega_{23} \Omega_{34}+\Omega_{13} \Omega_{32} \Omega_{24}\right)-\left(E-\bar{E}_{0}\right)\left(\Omega_{12} \Omega_{24}+\Omega_{13} \Omega_{34}\right)}{\operatorname{det}[E-\mathcal{H}]}
$$

One finds from Eq. (5.5) that $\tilde{b}_{4}(E)$ has four poles in the complex $E$-plane in the vicinity of the Landau level, which generate four resonance peaks in the reflection coefficient $\mathcal{R}(E)=$ $4 \Gamma_{1}\left|\tilde{b}_{4}(E)\right|^{2} \Gamma_{4}$. The interference effects appears in oscillations of the numerator in Eq. (5.5). Indeed, each term in the numerator corresponds to a certain path in a carrier inter-edge resonant transition, Fig. 3. These trajectories do interfere, since the phases of amplitudes $\Omega_{i j}$ are different. Using Eq. (4.13) we obtain

$$
\left|\tilde{b}_{4}(E)\right|=\frac{\left|\Omega_{12} \Omega_{24}\right|\left[\left(E-\bar{E}_{0}\right)\left(1+e^{2 \pi i B S / \phi_{0}}\right)-\left|\Omega_{23}\right|\left(e^{2 \pi i B S_{1} / \phi_{0}}-e^{2 \pi i B S_{2} / \phi_{0}}\right)\right]}{\operatorname{det}[E-\mathcal{H}]}
$$

where $S \equiv S_{1234}=\left(x_{3}-x_{2}\right)\left(y_{1}-y_{4}\right) / 2$ is the area enclosed by the two most probable trajectories of a carrier, moving from the impurities 1 to 4, Fig. 3. Correspondingly, $S_{1} \equiv$ $S_{123}$ and $S_{1} \equiv S_{234}$. If $S_{1}=S_{2}$ the second term in Eq. (5.6) is zero. Then the reflection coefficient vanishes when the flux $B S=n \phi_{0}$.

\section{B. Extended states near the Landau levels}

Let us assume that there exists only one most probable chain of non-diagonal resonant transitions in the Hamiltonian (5.1). Then neglecting all the $\Omega_{i j}$ in Eq. (5.1), which are not 
associated with this chain of transitions, one can rewrite the effective Hamiltonian as

$$
\mathcal{H}=\left(\begin{array}{cccccc}
\bar{E}_{0}-i \Gamma_{1} & \Omega_{21} & 0 & \cdots & 0 & 0 \\
\Omega_{12} & \bar{E}_{0} & \Omega_{32} & \cdots & 0 & 0 \\
0 & \Omega_{23} & \bar{E}_{0} & \cdots & 0 & 0 \\
\cdots & \cdots & \cdots & \cdots & \cdots & \cdots \\
0 & 0 & 0 & \cdots & \Omega_{n-1, n} & \bar{E}_{0}-i \Gamma_{n}
\end{array}\right)
$$

In this approximation it looks as the tunneling Hamiltonian for one-dimensional system with off-diagonal disorder. These systems were studied in many works, see for instance [17 21]. It was found that the localization length $L(E)$ diverges in the middle of the band $\left(E=\bar{E}_{0}\right)$, independent of the probability distribution of off-diagonal disorder [19]. In fact, this result does not necessarily imply the existence of an extended state at this energy. It was argued that the wave function would remain localized even in the middle of the band, but decaying away from the localization region only like an exponential of the square root of the distance [20,21]. In our case, however, the situation is different, since the sequence of non-diagonal elements in the Hamiltonian (5.7) is associated with the most probable path. Therefore, this sequence is not fully random, but is a subject of a certain constraint. (Similar physical situation has been discussed by Lifshitz and Kirpichenkov in their analysis of tunneling transparency of disordered systems [9]). As a result, the state in the middle of the band may appear as an extended one. Let us investigate the transport properties of our system in more detail.

Solving Eq. (5.4) with the Hamiltonian (5.7) we get for $\tilde{b}_{n}$

$$
\left|\tilde{b}_{n}(E)\right|=\frac{\left|\Omega_{12} \Omega_{23} \cdots \Omega_{n-1, n}\right|}{|\operatorname{det}[E-\mathcal{H}]|}
$$

The determinant in Eq. (5.8) can be rewritten in terms of minors and cofactors as

$$
\operatorname{det}[E-\mathcal{H}]=D_{n}(\mathcal{E})+i \Gamma_{1}^{+} D_{1, n}(\mathcal{E})+i \Gamma_{n}^{-} D_{n-1}(\mathcal{E})-\Gamma_{1}^{+} \Gamma_{n}^{-} D_{1, n-1}(\mathcal{E})
$$

where $\mathcal{E}=E-\bar{E}_{0}$ and $D(\mathcal{E})$ are the minor determinants of the matrix

$$
\mathcal{D}_{\alpha \beta}=\mathcal{E} \delta_{\alpha, \beta}+\Omega_{\alpha \beta} \delta_{\alpha-1, \beta}+\Omega_{\alpha \beta} \delta_{\alpha+1, \beta}
$$


so that $D_{n}=\operatorname{det}\left\{\mathcal{D}_{\alpha \beta}\right\}$ for $\alpha, \beta=1, \ldots, n, D_{1, n}=\operatorname{det}\left\{\mathcal{D}_{\alpha \beta}\right\}$ for $\alpha, \beta=2, \ldots, n, D_{n-1}=$ $\operatorname{det}\left\{\mathcal{D}_{\alpha \beta}\right\}$ for $\alpha, \beta=1, \ldots, n-1$, and $D_{1, n-1}=\operatorname{det}\left\{\mathcal{D}_{\alpha \beta}\right\}$ for $\alpha, \beta=2, \ldots, n-1$. Notice that the matrix $\{\mathcal{D}\}$ is obtained from the matrix $\{E-\mathcal{H}\}$ by setting $\Gamma_{1, n}=0$. All the determinants can be evaluated using the recursion relation

$$
D_{n}(\mathcal{E})=\mathcal{E} D_{n-1}(\mathcal{E})-\left|\Omega_{n-1, n}\right|^{2} D_{n-2}(\mathcal{E})
$$

where $D_{0}(\mathcal{E})=1$, and $D_{1}(\mathcal{E})=\mathcal{E}$. Finally one obtains for the probability of the inter-edge transition via $n$-impurities $\mathcal{R}_{n}(\mathcal{E}) \equiv \mathcal{R}(E)$, Eq. (5.2)

$$
\mathcal{R}_{n}(\mathcal{E})=4 \Gamma_{1} \frac{\left|\Omega_{12} \Omega_{23} \cdots \Omega_{n-1, n}\right|^{2}}{\left[D_{n}(\mathcal{E})-\Gamma_{1} \Gamma_{n} D_{1, n-1}(\mathcal{E})\right]^{2}+\left[\Gamma_{1} D_{1, n}(\mathcal{E})+\Gamma_{n} D_{n-1}(\mathcal{E})\right]^{2}} \Gamma_{n}
$$

It is clear that the $n$-dependence of $\mathcal{R}_{n}(\mathcal{E})$, determines the localization properties of electron states inside a sample. For instance, if these states are localized, $\mathcal{R}_{n}(\mathcal{E})$ decreases exponentially with $n$, and the longitudinal resistance vanishes. We can determine the localization length $L(\mathcal{E})$ by

$$
L^{-1}(\mathcal{E})=-\lim _{n \rightarrow \infty} \frac{1}{n} \ln \left|\mathcal{R}_{n}(\mathcal{E})\right|
$$

One can easily check that this definition of the localization length is essentially the same as the standard one in terms of eigenstates of the matrix $\{E-\mathcal{H}\}$ with $\Gamma_{1, n}$ in the Hamiltonian $\mathcal{H}$, Eq. (5.7), set to zero [19].

Consider Eq. (5.11) for $\mathcal{E}=0$ (the middle of band). One finds for $n=2 k+1$ that $D_{n}(0)=D_{n-2}(0)=0$, and

$$
\begin{aligned}
D_{1, n}(0) & =(-1)^{\frac{n-1}{2}}\left|\Omega_{23}\right|^{2}\left|\Omega_{45}\right|^{2} \cdots\left|\Omega_{n-1, n}\right|^{2} \\
D_{n-1}(0) & =(-1)^{\frac{n-1}{2}}\left|\Omega_{12}\right|^{2}\left|\Omega_{34}\right|^{2} \cdots\left|\Omega_{n-2, n-1}\right|^{2}
\end{aligned}
$$

Thus using Eq. (5.12) we find for the probability of the inter-edge transition

$$
\mathcal{R}_{n}(0)=\frac{4 \Gamma_{1} \Gamma_{n}}{\left(\Gamma_{1} \kappa_{n}^{-1}+\Gamma_{n} \kappa_{n}\right)^{2}}
$$

where 


$$
\kappa_{n}=\left|\frac{\Omega_{12} \Omega_{34} \cdots \Omega_{n-2, n-1}}{\Omega_{23} \Omega_{45} \cdots \Omega_{n-1, n}}\right|=\exp \left[\left(-\boldsymbol{r}_{12}^{2}+\boldsymbol{r}_{23}^{2}-\boldsymbol{r}_{34}^{2}+\cdots+\boldsymbol{r}_{n-1, n}^{2}\right) / 4 \ell^{2}\right]
$$

and $\boldsymbol{r}_{i j}^{2} \equiv\left(\boldsymbol{r}_{i}-\boldsymbol{r}_{j}\right)^{2}$. Similarly, for $n=2 k$ we obtain

$$
\mathcal{R}_{n}(0)=\frac{4 \Gamma_{1} \Gamma_{n} /\left|\Omega_{n-1, n}\right|^{2}}{\left(\kappa_{n-1}+\frac{\Gamma_{1} \Gamma_{n}}{\left|\Omega_{n-1, n}\right|^{2}} \kappa_{n-1}^{-1}\right)^{2}}
$$

By applying the central-limit theorem for random distribution of $\Omega_{i j}$ one finds from Eqs. (5.13), (5.15) and (5.17) that the localization length in the band center diverges,

$$
L^{-1}(0) \sim(1 / n)\left|\ln \kappa_{n}\right|=\frac{1}{n} \sum_{j}^{n}(-1)^{j} \frac{\boldsymbol{r}_{j-1, j}^{2}}{4 \ell^{2}} \sim \frac{1}{\sqrt{n}} \rightarrow 0
$$

Nevertheless, the electron states in the center of band remain localized, since $\mathcal{R}_{n}(0) \sim$ $\exp \left(-2 n^{1 / 2}\right) \rightarrow 0$ for $n \rightarrow \infty$, Eqs. (5.15), (5.17).

In our case, however, the distribution of $\Omega_{i j}$ in (5.7) is not totally random, since the effective Hamiltonian (5.7) contains only those non-diagonal amplitudes which generate interedge transitions with no appreciable attenuation. It follows from Eqs. (5.15), (5.17) that $\Omega_{i j}$ have to obey the requirement $\left|\kappa_{n}\right| \sim 1$, or $\sum_{j}(-1)^{j} \boldsymbol{r}_{j-1, j}^{2} \sim 4 \ell^{2}$, so that $\mathcal{R}_{n}(0)$ would remain of the order of one. We assume here that the above condition is fulfilled, and as a result the electron state at $\mathcal{E}=0$ is an extended one. Actually, this requirement is much weaker than that of Lifshitz and Kirpichenkov [9] for an appearance of the "resonance-percolation" trajectories in tunneling through disordered systems. The reason is that Lifshitz and Kirpichenkov looked for a band of extended states, and therefore they required approximately the same distance between impurities in the chain. In a contrast, our condition is a sufficient one only for a single extended state, where all other electron states are localized.

\section{Localization properties of electron states for $\mathcal{E} \neq 0$}

In order to investigate the electron transport along the chain of randomly distributed impurities, restricted by the constraint $\kappa_{n} \sim 1$, it is easier to start with the opposite case, 
where all impurities are aligned and therefore the non-diagonal amplitudes in the Hamiltonian (5.7) are the same, $\Omega_{i j}=\Omega$. Then it is rather obvious that the conducting band appears around $\mathcal{E}=0$. It can be seen immediately from Eq. (5.11) that in this case

$$
D_{n}(\mathcal{E})=\Omega^{n} \frac{\sin [(n+1) \alpha]}{\sin (\alpha)}
$$

where $\cos (\alpha)=\mathcal{E} / 2 \Omega$. Substituting Eq. (5.19) into Eq. (5.12) and taking for the simplicity $\Gamma_{1}=\Gamma_{n}=\Omega$ we obtain for probability of the inter-edge transition across a sample

$$
\mathcal{R}_{n}(\mathcal{E})=\frac{4 \Omega^{2}-\mathcal{E}^{2}}{4 \Omega^{2}-\mathcal{E}^{2} \cos ^{2}(n \alpha)}
$$

Therefore all the states for $|\mathcal{E}| \leq|\Omega|$ are indeed extended.

Now let us displace the impurity ' $k$ ' from its position so that $\Omega_{k-1, k} \neq \Omega$ and $\Omega_{k, k+1} \neq \Omega$. We assume for the definiteness that $\left|\Omega_{k-1, k}\right|,\left|\Omega_{k, k+1}\right| \ll|\Omega|$. As a result the impurity ' $k$ ' would generate the reflection of the Bloch waves inside the band. For the energy $\mathcal{E} \ll \Omega$, one finds that the situation resembles resonant tunneling through a double-barrier structure, namely the Bloch waves inside the band can pass the impurity ' $k$ ' only via the virtual state at $\mathcal{E}=0$. Then the probability of inter-edge transition can be written as

$$
\mathcal{R}_{n}^{(k)}(\mathcal{E})=\frac{4 \gamma_{k-1} \gamma_{k+1}}{\mathcal{E}^{2}+\left(\gamma_{k-1}+\gamma_{k+1}\right)^{2}}
$$

(c.f with Eq. (3.38)), where

$$
\begin{aligned}
& \gamma_{k-1}=2 \pi \varrho\left|\Omega_{k-1, k}\right|^{2}=\frac{\left|\Omega_{k-1, k}\right|^{2}}{|\Omega|} \\
& \gamma_{k+1}=2 \pi \varrho\left|\Omega_{k, k+1}\right|^{2}=\frac{\left|\Omega_{k, k+1}\right|^{2}}{|\Omega|}
\end{aligned}
$$

Here $\varrho=(2 \pi|\Omega|)^{-1}$ is the density of the band states.

The above result clarifies the meaning of the constraint $\kappa_{n} \sim 1$ which is the necessary one for the appearance of an extended state at $\mathcal{E}=0$. Indeed, the maximal value of the transition probability is reached at the resonance energy $\mathcal{E}=0$ and only for $\Omega_{k-1, k} / \Omega_{k, k+1}=1$, as always happened in the case of resonant scattering. Since all the other $\Omega_{i j}$ are the same, the above condition is equivalent to $\kappa_{n}=1$. The displacement of an another impurity would 
create the second double barrier structure along the chain. By approximating the total transmission probability as a product [19,22] $\mathcal{R}_{n}^{\left(k_{1}\right)} \mathcal{R}_{n}^{\left(k_{2}\right)}$, one finds that also in this case the maximal probability is reached for $\Omega_{k_{1}-1, k_{1}} / \Omega_{k_{1}, k_{1}+1}=1$ and $\Omega_{k_{2}-1, k_{2}} / \Omega_{k_{2}, k_{2}+1}=1$, so that $\kappa_{n}$ remains to be one. By continue this procedure of increasing disorder one finds that the resonance at $\mathcal{E}=0$ by itself would not provide the absence of an attenuation along the chain. One needs also to keep $\Omega_{k-1, k} / \Omega_{k, k+1}=1$, in an accordance with the requirement of $\kappa_{n} \sim 1$

Now let us estimate the transmission probability for $\mathcal{E} \neq 0$, by imposing $\Omega_{k-1, k}=\Omega_{k, k+1}$, so that the state $\mathcal{E}=0$ is the extended one. (It is quite clear from the above result that if the state $\mathcal{E}=0$ is localized, all the other states for $\mathcal{E} \neq 0$ are localized too). Using Eqs. (5.21), (5.22) we obtain

$$
\mathcal{R}_{n}(\mathcal{E})=\prod_{k} \frac{1}{\mathcal{E}^{2} \frac{|\Omega|^{2}}{4\left|\Omega_{k}\right|^{4}}+1}
$$

where $\Omega_{k-1, k}=\Omega_{k, k+1} \equiv \Omega_{k}$, Therefore the electron states for $\mathcal{E} \neq 0$ are localized with the localization length, Eq. (5.13)

$$
L^{-1}=C \ln \left(1+\frac{\mathcal{E}^{2}}{4\langle\Omega\rangle^{2}}\right) \sim \frac{\mathcal{E}^{2}}{\langle\Omega\rangle^{2}}
$$

and the transmission probability across a sample decreases exponentially with $n$, i.e. $\mathcal{R}_{n}(\mathcal{E}) \sim \exp \left[-\left(\mathcal{E}^{2} /\langle\Omega\rangle^{2}\right) n\right]$

Although we concentrated on electron transport inside the Landau mode $N=0$, it is clear that the results remain the same for any of Landau modes. Our analysis of the localization properties was based only on the off-diagonal disorder in the effective Hamiltonian (5.7) and on the constraint $\kappa_{n} \sim 1$, Eq. (5.16). Therefore the exponential is universal and the localization length near the Landau level $N$ band center can be written as

$$
L_{N}^{-1}(E) \sim\left(E-\bar{E}_{N}\right)^{2}
$$

where $\bar{E}_{N}=E_{N}+\epsilon_{N}$ and $\epsilon_{N}$ is the energy of the resonance near the Landau level $E_{N}$. Thus the critical energy $\bar{E}_{N}$ is shifted away from the exact center of the Landau level $E_{N}$ 
(as also follows from the numerical calculations [3,23]). Its position depends on the type of impurities in a sample: for attractive impurities it is below the Landau level, and above the Landau level for repulsive impurities.

We also like to point out that the peak value of the reflection coefficient $\mathcal{R}_{\max }=\mathcal{R}(0)$, Eqs. (5.15), (5.17), averaged over all possible resonant-percolation trajectories, determines the maximum of the total (two-terminal) resistance $\mathcal{G}^{-1}$, Eq. (2.12). Since our derivation of Eqs. (5.15),(5.17) does not depend on a particular Landau mode $N$, we expect that the peak value of two-terminal resistance remains the same for all Landau levels.

The particular behavior of the electron states near the the Landau level center, where one (resonance) level is extended and others are localized, is a consequence of the off-diagonal disorder of the effective Hamiltonian (5.7). The diagonal energy shift, due to resonance transitions between different impurities would violate the diagonal order. However, in a case of short range impurities the diagonal shift $\Delta_{j}$, Eq. (4.12), is small and can be can be neglected. When the potential range increases the renormalization of the resonance levels may be appreciable. Nevertheless, the diagonal disorder is determined by $\Delta_{j}-\langle\Delta\rangle$, where $\langle\Delta\rangle$, is the mean value of the diagonal energy shift, while the off-diagonal disorder, is determined by the overlap of the quasi-bound states near impurity sites, $\Omega_{i j}$. Since in strong magnetic field these states are concentrated near the impurity centers even for long range potentials, Eq. (3.52), one finds that $\Omega_{i j}$ would fluctuate much strongly than $\Delta_{j}-\langle\Delta\rangle$. In this case we may expect that the off-diagonal disorder would still play a dominant role in a determination of localization properties of electron states.

Finally we would like to mention that in the case of more than one resonant-percolation trajectories connect the same initial and final impurity sites (as in Eq.(5.3)) (self-crossing trajectories), one cannot re-arrange the effective Hamiltonian in a form of the off-diagonal nearest-neighbor coupling. In this case the Aharonov-Bohm interference-effects may play an essential role and the value of the exponential in Eq. (5.25) can be modified. This effect needs a special investigation and is not a subject of this paper. 


\section{SUMMARY}

In this paper we presented a systematic study of the resonant scattering on impurities of $2 \mathrm{D}$ electron gas in the presence of a strong magnetic field. It follows from our analysis that this process can play a dominant role in delocalization of the electron states near the Landau levels.

Starting with a detailed analysis of the resonant scattering on a single impurity we demonstrated that any no matter how weak impurity generates resonance states in the vicinity of the Landau levels: an attractive impurity produces the resonances below the Landau levels, and a repulsive impurity - above the Landau levels. Our method can be applied to any type of impurities. In particular, we found simple analytical expressions for the resonance energy and the partial widths for any short range impurity and for long range impurity of a Gaussian type. Although these expressions look rather similar for attractive and repulsive impurities, the mechanism of the inter-edge transition is quite different: The attractive impurity gives rise to the inter-edge transitions via coupling with the resonant state in the nearest non-propagating Landau mode. In contrast, the inter-edge transition generated by a repulsive impurity takes place in the same propagating Landau mode. As a result, the transition probability via a repulsive impurity is in general larger than that produced by an attractive impurity. For instance, in the case of an attractive impurity the value of the resonance peak in the longitudinal resistance drops down very rapidly, when the impurity is away from the center of a sample. On the other hand, the value of the resonance peak generated by a repulsive impurity is less sensitive to the impurity position.

In the case of wide samples the inter-edge transport is generated by a multiple scattering of carriers on different impurities. It is known that in the absence of magnetic field the

electron states of disordered 2D system are localized. Therefore the question is of how a strong magnetic field can delocalize the states, so that the inter-edge current could flow across a sample. Our analysis of the resonant scattering on impurities suggests the following reasons. First, each impurity in a strong magnetic field generates the resonance at the same 
energy which depends only on the type of impurity. The imaginary part of the resonance energy (the width) is appreciable only for those impurities which are close to the sample edges, and it is negligible for impurities in the bulk. Second, the quasi-bound states are strongly localized near the impurity center (even for a long range impurity, as follows from our analysis of the Gaussian-type impurity). As a result, the diagonal energy shift plays no essential role in the transport properties of carriers, in comparison with the off-diagonal transition amplitudes. (In a case of short range impurities the diagonal energy shift can be neglected at all). Therefore the system resembles eventually the one with off-diagonal disorder.

The analysis of 1D system with the off-diagonal disorder shows that the localization properties of the level in the center of the band (the resonance energy) differ from that of the other states. One finds that the wave function of this state is localized, but it decays only like an exponent of the square root of the distance. It is enough to impose a weak constraint on the position of impurities in the chain in order to delocalize this state. In our case of 2D disordered system, the transport takes place along the most probable trajectories (the resonant percolation trajectories). If we neglect the contributions from self-crossing trajectories, the problem is reduced to that of the 1D system with off-diagonal disorder. The requirement for the resonance-percolation trajectory in $2 \mathrm{D}$ system delocalizes the level in the center of the band. We found this loose constraint on the impurity positions in the chain, and demonstrated that all other levels except for the central one, remain localized. The analysis of the localization properties can be done analytically. We estimated the behavior of the localization length near the band center under the above constraint on the impurity positions, and obtain the square power-like divergence of the localization. The latter would remain the same for any of Landau levels, as well as the peak value of the two-terminal resistance.

It is clear that this analysis can give only an estimation of the critical exponent. For instance, the neglected contribution of the self-crossing resonant-percolation trajectories may change the value of this exponent. These and other effects are not considered here and need 
further investigation.

\section{DISCUSSION}

It is known that all the states are localized for a 2D electronic system with arbitrary amount of disorder. However, when a uniform magnetic field is applied perpendicular to the system, there is an indication that extended electron states exist at only one energy within the broadened Landau level. Other states should be localized. Despite various attempts the nature of the delocalization is not clear yet. In Section 5 we proposed a possible mechanism of the delocalization and predicted universal power-law divergence of the localization length. Therefore it is interesting to compare our results with the existing approaches.

Two different theories exist which predict the universal power-low divergence $\left(E-\bar{E}_{N}\right)^{-\nu}$ of the localization length for noninteracting electrons in the presence of a high magnetic field. The first based on a classical percolation theory, considers the extreme high-magnetic-field limit when the magnetic length is much less than the correlation length of the disorder potential [24]. This approach predicts the value of the critical exponent $\nu=4 / 3$. The second theory is based on an approximate reduction of the problem to nonlinear $\sigma$-model [25]. This model is applicable when the magnetic length is much larger than the correlation length of the disorder potential. The value of the critical exponent, however, is not known for the non-linear $\sigma$-model.

Existing numerical calculations also establish the power-low divergence of the localization length. The value of $\nu \approx 2.2-2.3$ was obtained for the lowest Landau level in the case of short-range white-noise random potential [26] and for $\delta$-function or Gaussian-type disorder [3]. Still, the numerical analysis does not illuminate the origin of the localization mechanism.

Our analytical study of this problem given for randomly distributed identical impurities, also demonstrates the appearance of a delocalized state and the universal power-low divergence of the localization length with $\nu \approx 2$. An important advantage of our approach is that

it clearly displays the delocalization mechanism. Paradoxically, the extended state appears 
as a result of the localization of an electron near impurity sites due to strong magnetic field. Since the energy of these localized states is the same for all impurities, the disorder takes place only in non-diagonal matrix elements (2D off-diagonal disorder). Then as explained in Section 5, the resonant percolation trajectories appear which lead to a delocalization of one particular level. In fact, one can prove the existence of these trajectories even for an arbitrary small density of impurities, providing the size of a sample is large enough. The proof will be given in a separate publication as well as an extension for a general case of random disorder (non identical impurities).

Since we considered here the case of identical impurities, the position of the critical energy $\bar{E}_{N}$ is slightly shifted from the center of the corresponding Landau level, $E_{N}$ (c.f. with finding in [3,23]). It lies in an agreement with the result of [25], which predicts only the universality of the exponent, but not the critical energy. The latter can be slightly changed, depending on properties of a sample.

The scaling behavior of the localization length is closely related to the remarkable temperature dependence of the half-width of peaks in the longitudinal resistance. The experiments of Wei et. al. [27 showed that this quantity vanishes as $\sim T^{\alpha}$, where $\alpha \approx 0.42$ is the same for the integer and for the fractional QHE. Despite the fact that $\alpha$ is believed to involve the inelastic scattering length, which is not understood in the high-field regime, the universality of $\alpha$ indicates on the same localization mechanism for the integer and the fractional QHE. Indeed it was explicitly established by using the composite fermions [28,29] or the anyons [30] approaches to the fractional QHE. It is rather clear that in this framework our approach would also predict the same localization mechanism for the integer and the fractional QHE, except for half-filled Landau level. In the latter case the system would be equivalent to the spinless fermions in zero magnetic field [31]. Hence, it cannot be effectively reduced to one described in terms of off-diagonal 2D disorder, and therefore our analysis would not be more valid. 


\section{ACKNOWLEDGMENTS}

I owe special thanks to A. Aronov for useful discussions and important suggestions. I am also grateful to A. Finkel'stein, Y. Gefen, S. Iordansky, Y. Levinson and A. Kamenev for useful discussions.

\section{APPENDIX A: SQUARE WELL CONFINED POTENTIAL IN THE MAGNETIC FIELD}

Consider Eq. (2.3) for the Landau-mode wave functions in the confining potential: $W(y)=0$ for $|y| \leq a$ and $W(y)=+\infty$ for $|y|>a$. The general solution of this equation can be written as a linear combination of parabolic cylinder functions, $D_{\nu}(z)$ and $D_{\nu}(-z)$,

$$
\Phi_{n k}(y)=C_{+} D_{\nu}(z)+C_{-} D_{\nu}(-z)
$$

where $\hbar \omega_{c}(\nu+1 / 2)=E_{n k}$ and $z=\sqrt{2}\left(y-\ell^{2} k\right) / \ell$.

$$
\begin{aligned}
D_{\nu}(z)=\frac{2^{\nu / 2} e^{-z^{2} / 4}}{\sqrt{\pi}} & {\left[\Gamma\left(\frac{1+\nu}{2}\right) \cos (\pi \nu / 2){ }_{1} F_{1}\left(-\frac{\nu}{2} ; \frac{1}{2} ; \frac{z^{2}}{2}\right)\right.} \\
& \left.+\sqrt{2} z \Gamma\left(1+\frac{\nu}{2}\right) \sin (\pi \nu / 2){ }_{1} F_{1}\left(\frac{1-\nu}{2} ; \frac{3}{2} ; \frac{z^{2}}{2}\right)\right]
\end{aligned}
$$

Here ${ }_{1} F_{1}$ is the confluent hypergeometric (or Kummer) function. For large positive $z \gg 1$ and $z \gg|\nu|$

$$
D_{\nu}(z) \sim e^{-z^{2} / 4} z^{\nu}\left[1-\frac{\nu(\nu-1)}{2 z^{2}} \pm \cdots\right]
$$

and if $z$ is large and negative $z \ll-1$ and $z \ll-|\nu|$

$$
\begin{aligned}
D_{\nu}(z) \sim e^{-z^{2} / 4} z^{\nu} & {\left[1-\frac{\nu(\nu-1)}{2 z^{2}} \pm \cdots\right] } \\
& -\frac{\sqrt{2 \pi}}{\Gamma(-\nu)} e^{\nu \pi i} e^{z^{2} / 4} z^{-\nu-1}\left[1+\frac{(\nu+1)(\nu+2)}{2 z^{2}} \pm \cdots\right]
\end{aligned}
$$

By supplementing the boundary conditions, $\Phi_{n k}( \pm a)=0$ one obtains the energy spectrum from the equation 


$$
D_{\nu}\left(z_{+}\right) D_{\nu}\left(z_{-}\right)-D_{\nu}\left(-z_{+}\right) D_{\nu}\left(-z_{-}\right)=0
$$

where $z_{ \pm}=(a / \ell) \pm k \ell$. Let us consider the region of $\left|z_{ \pm}\right| \gg 1$, where one can use the asymptotic representation of the parabolic cylinder functions. Substituting Eqs. (A3), (A4) into Eq. (A5), and keeping only the leading terms of the expansion, one obtains

$$
z_{+}^{2 \nu+1} e^{-z_{+}^{2} / 2}+z_{-}^{2 \nu+1} e^{-z_{-}^{2} / 2}=\sqrt{\frac{2}{\pi}} \frac{1-e^{-2 \pi i \nu}}{2 i} \Gamma(1+\nu)
$$

Taking $\nu=n+\alpha_{n}$ and expanding Eq. (A6) in power of $\alpha_{n}$ we get

$$
\alpha_{n}=\frac{2^{n}}{\sqrt{\pi} n !}\left[\left(\frac{a}{\ell}-k \ell\right)^{2 n+1} \exp \left(-\frac{\left(a-k \ell^{2}\right)^{2}}{\ell^{2}}\right)+\left(\frac{a}{\ell}+k \ell\right)^{2 n+1} \exp \left(-\frac{\left(a+k \ell^{2}\right)^{2}}{\ell^{2}}\right)\right]
$$

Using $\mathcal{K}_{n}(k)=\hbar \omega_{c} \alpha_{n}$, we arrive to Eq. (2.6) for the energy spectrum in the confined square-well potential for $\left|a \pm k \ell^{2}\right| \gg \ell$. One also finds that the eigenfunctions, Eq. (A1), are well approximated by by the harmonic oscillator wave functions, Eq. (2.5), in the region $\left|a \pm k \ell^{2}\right| \gg \ell$.

\section{APPENDIX B: HOPPING TRANSITIONS BETWEEN TWO IMPURITIES}

Consider two impurities, each of them generates a bound state with the energy $E_{1}$ and $E_{2}$ respectively

$$
\begin{aligned}
& \left(\mathcal{K}+V_{1}\right) \psi_{1}=E_{1} \psi_{1} \\
& \left(\mathcal{K}+V_{2}\right) \psi_{2}=E_{2} \psi_{2}
\end{aligned}
$$

Here $\mathcal{K}$ denotes the kinetic energy term and $V_{1,2}$ are the impurity potentials. We assume that $E_{1} \sim E_{2}$. These energies are obviously not the eigenvalues of the whole Hamiltonian, when the two potentials are "switched on". The exact energies are obtained from the the Schroedinger equation

$$
\left(\mathcal{K}+V_{1}+V_{2}\right) \psi=E \psi
$$

Let us rewrite this equation in the integral form as 


$$
\psi=\psi_{1}+\tilde{G}_{1} V_{2} \psi=\psi_{1}+\tilde{G} V_{2} \psi_{1}
$$

where the Green's functions

$$
\tilde{G}_{1}=\frac{Q_{1}}{E-\mathcal{K}-V_{1}}
$$

and

$$
\tilde{G}=\tilde{G}_{1}+\tilde{G}_{1} V_{2} \tilde{G}
$$

Here $Q_{1}$ is the projection operator which excludes the state $\left|\psi_{1}><\psi_{1}\right|$ from the spectral representation of $G_{1}=\left(E-\mathcal{K}-V_{1}\right)^{-1}$. The bound state energy $E$ is given by

$$
E=E_{1}+<\psi_{1}\left|V_{2}\right| \psi_{1}>+<\psi_{1}\left|V_{2} \tilde{G} V_{2}\right| \psi_{1}>
$$

Let us assume that the impurities are separated far enough so that the wave functions $\psi_{1,2}$ are small in the regions of the potentials $V_{2,1}$, respectively. Then one can approximate $Q_{1} \cong 1$ in the region of the potential $V_{2}$, and therefore $V_{2} \tilde{G} V_{2} \cong V_{2} G V_{2}$, where

$$
G=\frac{1}{E-\mathcal{K}-V_{1}-V_{2}}=G_{2}+G_{2} V_{1} G_{2}+G_{2} V_{1} G_{2} V_{1} G_{2}+\cdots
$$

is the total Green's function, and $G_{2}=\left(E-\mathcal{K}-V_{2}\right)^{-1}$. Using the spectral representation of the Green's function $G_{2}$ and Eq. (B) we get for $G_{2}$ and $G$ in the energy region $E \sim E_{1,2}$

$$
\begin{gathered}
G_{2} \rightarrow \frac{\left|\psi_{2}><\psi_{2}\right|}{E-E_{2}} \\
G \rightarrow \frac{\left|\psi_{2}><\psi_{2}\right|}{E-E_{2}-<\psi_{2}\left|V_{1}\right| \psi_{2}>}
\end{gathered}
$$

Substituting this result into Eq. (B6) we get

$$
E=E_{1}+<\psi_{1}\left|V_{2}\right| \psi_{1}>+\frac{<\psi_{1}\left|V_{2}\right| \psi_{2}><\psi_{2}\left|V_{2}\right| \psi_{1}>}{E-E_{2}-<\psi_{2}\left|V_{1}\right| \psi_{2}>}
$$

This equation can be identically rewritten as

$$
\operatorname{det}\left(\begin{array}{cc}
E-E_{1}-\Delta_{1} & \Omega \\
\Omega & E-E_{2}-\Delta_{2}
\end{array}\right)=0
$$


where $\Delta_{1}=<\psi_{1}\left|V_{2}\right| \psi_{1}>$ and $\Delta_{2}=<\psi_{2}\left|V_{1}\right| \psi_{2}>$ are the diagonal energy shifts, and $\Omega=<\psi_{1}\left|V_{2}\right| \psi_{2}>$ is the hopping transition amplitude between two impurities. Using the Schroedinger equation one obtains useful formula for the hopping amplitude

$$
\Omega=<\psi_{1}\left|V_{2}\right| \psi_{2}>\cong<\psi_{1}\left|V_{1}\right| \psi_{2}>\cong<\psi_{1}|\bar{E}-\mathcal{K}| \psi_{2}>
$$

where $\bar{E}=\left(E_{1}+E_{2}\right) / 2$. Notice that $\Omega \gg \Delta_{1,2}$. 


\section{REFERENCES}

[1] P. Streda, J. Kucera, and A.H. MacDonald, Phys. Rev. Lett. 59, 1973 (1987).

[2] M. Büttiker, Phys. Rev. B38, 9375 (1988).

[3] D. Liu and S.D. Sarma, Phys. Rev. B49, 2677 (1994), and references therein.

[4] Y. Avishai, M.Ya. Azbel and S.A. Gredeskul, Phys. Rev. B48, 17280 (1993); M.Ya. Azbel, Phys. Rev. B49, 5463 (1994), and references therein.

[5] R.E. Prange, Phys. Rev. B9, 4802 (1981).

[6] J.K. Jain and S.A. Kivelson, Phys. Rev. Lett. 60, 1542 (1988).

[7] V.L. Pokrovskiı, L.P. Pryadko, and A.L. Talapov Sov. Phys. JETP, 68, 376 (1988).

[8] E.V. Sukhorukov, M.I. Lubin, C. Kunze and Y.B. Levinson, Phys. Rev. B, in press.

[9] I.M. Lifshitz and V.Ya. Kirpichenkov, Sov. Phys. JETP 50, 499 (1979).

[10] We are not entering into the question of how to obtain the longitudinal and the Hall resistance from $\mathcal{G}$. This problem has been extensively discussed in the literature. For instance, one can use the Büttiker formalism [2].

[11] S.A. Gurvitz and Y.B. Levinson, Phys. Rev. B47, 10578 (1993).

[12] S.A. Gurvitz and G. Kalbermann, Phys. Rev. Lett. 59, 262 (1987); S.A. Gurvitz, Phys. Rev. A38, 1747 (1988).

[13] A.I. Larkin and K.A. Matveev, Sov. Phys. JETP 66, 580 (1987).

[14] D. Sokolovski, Phys. Lett. A132, 381 (1988).

[15] S.A. Gurvitz, Phys. Rev. B44, 11924 (1991).

[16] A.M. Frishman and S.A. Gurvitz, Phys. Rev. B47, 16348 (1993).

[17] F.J. Dyson, Phys. Rev. 92, 1331 (1953). 
[18] I.M. Lifshitz, Sov. Phys. Usp. 7, 549 (1964).

[19] G.T. Theodorou and M. Cohen, Phys. Rev. B13, 4597 (1976).

[20] A. Bovier, Journal of Stat. Phys. 56, 645 (1989), and references therein.

[21] M. Inui, S.A. Trugman and E. Abrahams, Phys. Rev. B49, 3190 (1994).

[22] R.L. Bush, Phys. Rev. B6, 1182 (1972).

[23] H. Aoki, J. Phys. C10, 2583 (1977).

[24] S.A. Trugman, Phys. Rev. B27, 7539 (1983), and references therein.

[25] A.M.M. Pruisken, Phys. Rev. Lett. 61, 1297 (1988), and references therein.

[26] B. Huckestein and B. Kramer, Phys. Rev. Lett. 64, 1437 (1990).

[27] H.P. Wei, D.C. Tsui, M. Paalanen, and A.M.M. Pruisken, Phys. Rev. Lett. 61, 1294 (1988).

[28] J.K. Jain, Phys. Rev. Lett. 63, 199 (1990).

[29] J.K. Jain, S.A. Kivelson, and N. Trivedi, Phys. Rev. Lett. 64, 1297 (1990).

[30] D. Schmeltzer, Phys. Rev. B46, 1591 (1992).

[31] B.I. Halperin, P.A. Lee and N. Read Phys. Rev. B47, 7312 (1993). 


\section{FIGURES}

FIG. 1. (a) Energy spectrum for 2D electron gas in a strong magnetic field for a rectangular confining potential. (b) Effective 'kinetic energy' for different Landau bands.

FIG. 2. Schematical illustration of the classical hopping motion along the boundaries of a sample and the inter-edge transitions via different impurities.

FIG. 3. Different optimal trajectories for the inter-edge transition via four impurities. 